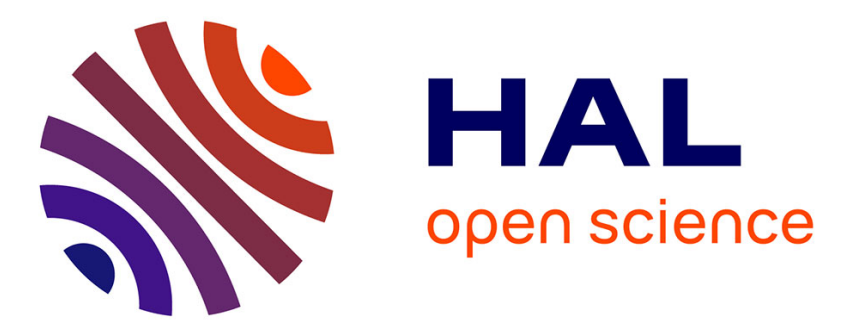

\title{
Robust Consensus of High-Order Systems under Output Constraints: Application to Rendezvous of Underactuated UAVs
}

Esteban Restrepo, Antonio Loria, Ioannis Sarras, Julien Marzat

\section{- To cite this version:}

Esteban Restrepo, Antonio Loria, Ioannis Sarras, Julien Marzat. Robust Consensus of High-Order Systems under Output Constraints: Application to Rendezvous of Underactuated UAVs. IEEE Transactions on Automatic Control, 2023, 68 (1), pp.329-342. 10.1109/TAC.2022.3144107 . hal-03275331v2

\section{HAL Id: hal-03275331 \\ https://hal.science/hal-03275331v2}

Submitted on 5 Jan 2022

HAL is a multi-disciplinary open access archive for the deposit and dissemination of scientific research documents, whether they are published or not. The documents may come from teaching and research institutions in France or abroad, or from public or private research centers.
L'archive ouverte pluridisciplinaire $\mathbf{H A L}$, est destinée au dépôt et à la diffusion de documents scientifiques de niveau recherche, publiés ou non, émanant des établissements d'enseignement et de recherche français ou étrangers, des laboratoires publics ou privés. 


\title{
Robust Consensus of High-Order Systems under Output Constraints: Application to Rendezvous of Underactuated UAVs
}

\author{
Esteban Restrepo Antonio Loría Ioannis Sarras Julien Marzat
}

\begin{abstract}
We solve output- and state-consensus problems for multi-agent high-order systems in feedback form. We consider systems interconnected over arbitrary (connected) undirected-topology networks as well as directed spanningtrees and directed cycles. We assume that the systems may be subject to multiple restrictions in the form of output or state constraints, such as limited-range measurements, physical limitations, etc. In addition, we suppose that the systems may be subject to external disturbances. Under these conditions, we present a control framework and a formal analysis that establishes robust stability in the input-to-state sense. The former relies on a modified backstepping method and the latter on multi-stability theory. Finally, we apply our approach to a case-study of interest in the aerospace industry: safetyaware rendezvous control of underactuated UAVs subject to connectivity and collision-avoidance constraints.
\end{abstract}

Index Terms-Consensus, multi-agent vehicles, control under constraints, robust stability, Lyapunov methods.

\section{INTRODUCTION}

The literature on consensus control, which constitutes the basis of cooperative interaction for multi-agent systems, is rife with works addressing diverse problems for a variety of dynamical systems, both linear [1]-[3] and nonlinear - most often second-order mechanical systems [4]-[8], but consensus of high-order systems is addressed, e.g., in [9]-[12].

While many articles address consensus problems for fairly generic classes of systems, the latter may fall short at representing many meaningful and complex engineering problems. A good example of a scenario of cooperative systems in which a plethora of difficulties appear naturally is that of rendezvous control of unmanned-autonomous vehicles (UAVs). For a start, the systems' dynamics is nonlinear and underactuated, so the literature on consensus tailored for linear low-order

Manuscript in press. Received April 21, 2021, revised October 27, 2021, accepted December 27, 2021. The work of A. Loría was supported in part by the French ANR via project HANDY, contract number ANR-18-CE40-0010 and by CEFIPRA under the grant number 6001-A. Recommended by Associate Editor C. Kellett. (Corresponding author: Esteban Restrepo.)

Esteban Restrepo, during the elaboration of this work, was with DTIS, ONERA, Univ Paris-Saclay, F-91123 Palaiseau, France (e-mail: este.restrepo95@gmail.com).

Antonio Loría is with Lab des signaux et systèmes, CNRS, 91190, Gif sur Yvette, France (e-mail: antonio.loria@cnrs.fr).

loannis Sarras is with DTIS, ONERA, Univ Paris-Saclay, F-91123 Palaiseau, France (e-mail: ioannis.sarras@onera.fr).

Julien Marzat is with DTIS, ONERA, Univ Paris-Saclay, F-91123 Palaiseau, France (e-mail: julien.marzat@onera.fr). systems does not apply. In addition, the measurements often come from embedded relative-measurement sensors, which are reliable only if the vehicles remain within a limited range. This translates into guaranteeing that the UAVs do not drift "too far" apart from their neighbors. Moreover, the use of such sensors naturally imposes directed network topologies. Furthermore, autonomous vehicles moving "freely" in the workspace are prone to undesired collisions. Finally, UAVs are constantly subject to external undesired forces generated, e.g., by wind gusts, aerodynamic effects or unmodeled dynamics. These constitute external disturbances at different levels in the dynamic model.

The aspects previously described coin a realistic scenario of automatic control of multi-agent systems that is not restricted to UAVs. Consensus under such conditions has been addressed in the literature, but, to the best of our knowledge, never simultaneously. This is the core contribution of this paper.

Many articles address the constrained consensus problem for low-order systems interconnected over, both, undirected and directed topologies [13]-[16]. Fewer works, however, address the consensus control problem with constraints for high-order systems. In [17] a tracking consensus controller is proposed for networked systems over undirected graphs only, and the constraints are considered on the synchronization error and not directly on the inter-agent relative states. In [18] a synchronization controller is designed using the prescribedperformance framework in order to achieve consensus over directed graphs with desired bounds on the transient response. Nevertheless, as in [17], the prescribed-performance constraints are imposed on the consensus error and not on the inter-agent relative states. A consensus control for highorder systems with constraints and interacting over strongly connected directed graphs is presented in [19]. Yet, the constraints considered therein weigh on each individual agent's states (e.g., constraints on the velocity, the acceleration, etc.), and do not reflect inter-agent restrictions. Model-predictivecontrol algorithms are proposed in [20] and [21] for consensus of high-order systems, but only constraints on the decoupled states of the agents or only collision avoidance is considered and robustness is not investigated. Robustness is addressed in [22], but for linear systems without inter-agent constraints.

The main contribution of this paper consists in a control framework for consensus of high-order nonlinear systems in feedback form, under inter-agent constraints. Our main 
statements apply to multi-agent systems interconnected over arbitrary connected undirected graphs, directed spanning trees, and directed cycles. Directed spanning-tree and directed-cycle topologies present practical interests of their own as they appear naturally in leader-follower configurations [7] and in the context of cyclic pursuit [23].

To guarantee the systems' safety as well as the integrity of the topology through the maintenance of connectivity, our controllers guarantee that output inter-agent constraints are respected. To achieve this, the said constraints are encoded in control laws that are derived as the gradients of barrier Lyapunov functions [24]. Essentially, the control input is defined as a function of the state that grows unboundedly as the system's state approaches a specified region. This technique is reminiscent of potential/navigation functions used, e.g., in robot control at least since the seminal work [25].

Our control framework is tailored for the fairly large class of systems in feedback form. Hence, the control architecture also relies on the popular passivity-based backstepping method. However, since in the presence of constraints this may involve successive derivatives of functions with multiple saddle points, we use the refined command-filtered backstepping approach [26], in which the successive derivatives are approximated by linear strictly-positive-real filters.

Beyond the control design, a significant part of our contribution resides in the formal analysis. In that regard, we stress that our method is based on the edge-agreement framework introduced in [27], by which the consensus problem is recast as one of stabilization of the origin. Now, since inter-agent constraints may introduce undesired unstable equilibria in the edges' space, the closed-loop system is analyzed using tools from [28] and [29] for multi-stable systems. Specifically, we establish attractivity of the consensus manifold as well as robustness with respect to external disturbances, in the sense of practical-input-to-state stability. This property is stronger than mere convergence, more often found in the literature.

Finally, to demonstrate the utility of our main theoretical findings, we show how our control framework may be applied to the rendezvous control problem for a group of underactuated UAVs, subject to connectivity and collision avoidance constraints, using only local measurements. This problem is relevant to the aerospace industry, in view of the increasing interest for safety-aware fleet deployment. It was addressed, e.g., in [30]-[33] for swarms of underactuated UAVs interconnected through undirected and directed communication topologies, but none of these works addresses the problem under inter-agent constraints. Connectivity constraints are considered in [34], but only for UAVs interconnected under undirected topologies and collision-avoidance is not addressed. In turn, the latter is considered in [35], but only for systems interconnected under an undirected topology.

In Section II we describe the problems that we address, in Section III we present our main statements, in Section IV we present a formal stability analysis, in Section $\mathrm{V}$ we present our novel results on formation consensus of UAVs, and the paper is wrapped up with some concluding remarks in Section VI.

\section{MOdel AND PROBLEM FORMULATION}

We consider multi-agent nonlinear systems in feedback normal form, of high relative degree with respect to an output of interest and subject to additive disturbances. More precisely, we consider $N$ multi-variable systems of relative degree $\rho$ defined by the equations

$$
\begin{aligned}
\dot{x}_{i, l} & =x_{i, l+1}+\theta_{i, l}(t), \quad l \leq \rho-1 \\
\dot{x}_{i, \rho} & =u_{i}+\theta_{i, \rho}(t),
\end{aligned}
$$

where $x_{i, l} \in \mathbb{R}^{n}, l \leq \rho, i \leq N$, denotes the components of the state of each agent, $u_{i} \in \mathbb{R}^{n}$ is the control input, $\theta_{i, l}$ : $\mathbb{R}_{\geq 0} \mapsto \mathbb{R}^{n}$ is an essentially bounded function that represents a disturbance, and $x_{i, 1}$ is considered an output of interest.

The class of systems with model (1) is fairly wide. This set of equations may represent, e.g., linearized nonlinear systems via feedback and coordinate transformation, such as secondorder feedback linearizable systems in which the outputconsensus problem consists in driving all the positions $x_{i, 1}$ to a common constant value and all the velocities $x_{i, 2}$ to zero. The model (1) may also represent nonlinear systems under a coordinate transformation and a preliminary dynamic control feedback loop, in which case some of the states are the controller's — see, e.g., Section V.

In terms of the systems' interaction, it is assumed that each agent has access only to local information from a limited number of neighbors. The latter is represented by a graph, denoted $\mathcal{G}=(\mathcal{V}, \mathcal{E})$, where the set of nodes $\mathcal{V}:=\{1,2, \ldots, N\}$ corresponds to the labels of the agents and the set of edges $\mathcal{E} \subseteq \mathcal{V}^{2}$, of cardinality $M$, represents the communication between a pair of nodes - an edge $e_{k}, k \leq M$, is an ordered pair $(i, j) \in \mathcal{E}$ indicating that agent $j$ has access to information from node $i$, via measurement or communication. We refer to an initial graph as the graph corresponding to the existing edges at $t=0$ and we pose the following.

Standing Assumption: the initial graph is either undirected and connected, or a directed spanning tree or a directed cycle.

Furthermore, we consider that the multi-agent system is subject to inter-agent constraints that may be defined as a set of restrictions on the system's output. Without loss of generality, let the first component $x_{i, 1}$ be the output for the $i$ th agent, with $i \leq N$, and define the relative-output state as

$$
z_{k, 1}:=x_{i, 1}-x_{j, 1} \quad \forall k \leq M, \quad(i, j) \in \mathcal{E} .
$$

For each $k \leq M$, let $\Delta_{k}$ and $\delta_{k}$, satisfying $0 \leq \delta_{k}<\Delta_{k}$, be the upper and minimal distances, respectively and let $|\cdot|$ denote the Euclidean norm. Then, the set of inter-agent output constraints is defined as

$$
\mathcal{D}:=\left\{z_{1} \in \mathbb{R}^{n M}: \delta_{k}<\left|z_{k, 1}\right|<\Delta_{k}, \forall k \leq M\right\} .
$$

The control goal is for the agents to achieve output consensus with a non-zero displacement, centered at a point of nonpredefined coordinates (as for instance in a formation control problem), under the constraint that the output trajectories must remain in the set $\mathcal{D}$ at all times. Furthermore, besides achieving the output-consensus goal it is additionally required to achieve consensus of a number of states $r \leq \rho$. This is useful, 
for instance, in the context of flocking in formation, attitude consensus, etc.

Mathematically, the output-consensus problem translates into making $x_{i, 1}-x_{j, 1} \rightarrow z_{k, 1}^{d}$, or equivalently, $z_{k, 1} \rightarrow z_{k, 1}^{d}$ in the relative coordinates, where $z_{k, 1}^{d} \in \mathbb{R}^{n}$ denotes the desired relative state between a pair of neighboring agents $i$ and $j$. Moreover, akin to (2), define the relative states

$$
z_{k, l}:=x_{i, l}-x_{j, l}, \quad \forall k \leq M, \quad \forall l \in\{2, \ldots, r\} .
$$

Then, the problem of consensus of the high-order states corresponds to making $z_{k, l} \rightarrow 0$, for all $l \in\{2, \ldots, r\}$.

Our control approach to solve this problem relies on the edge-agreement framework for modeling of graphs [27]. In this framework the variables defined in (2) denote the states of the interconnection arcs in the graph, instead of those of the nodes, which are more commonly used. This has the advantage of recasting the consensus objective as the stabilization of the origin in error coordinates.

To describe the setting, we start by recalling some notations and concepts. We denote the so-called incidence matrix of a graph by $E \in \mathbb{R}^{N \times M}$, which is a matrix with rows indexed by the nodes and columns indexed by the edges. Its $(i, k)$ th entry is defined as follows: $[E]_{i k}:=-1$ if $i$ is the terminal node of edge $e_{k},[E]_{i k}:=1$ if $i$ is the initial node of edge $e_{k}$, and $[E]_{i k}:=0$ otherwise. Let $x_{l}^{\top}=\left[x_{1, l}^{\top} \cdots x_{N, l}^{\top}\right] \in \mathbb{R}^{n N}$ be the collection of each state of all the agents of the system, for all $l \leq \rho$. Then, the edge states in (2) and (4) satisfy

$$
z_{l}:=\left[E^{\top} \otimes I_{n}\right] x_{l}, \quad l \in\{1, \ldots, r\} .
$$

where $z_{l}^{\top}=\left[z_{1, l}^{\top} \cdots z_{M, l}^{\top}\right] \in \mathbb{R}^{n M}$, ' $\otimes$ ' denotes the Kronecker product, and $I_{n}$ the identity matrix of dimension $n \times n$. Also, we introduce the error variable

$$
\tilde{z}_{1}=\left[E^{\top} \otimes I_{n}\right] x_{1}-z_{1}^{d},
$$

where $z_{1}^{d \top}=\left[z_{1,1}^{d \top} \cdots z_{M, 1}^{d \top}\right] \in \mathbb{R}^{n M}$. Then, in the error edge coordinates, the consensus objective is that

$$
\begin{array}{ll}
\lim _{t \rightarrow \infty} \tilde{z}_{1}(t)=0 & \\
\lim _{t \rightarrow \infty} z_{l}(t)=0, & \forall l \in\{2, \ldots, r\} \\
\lim _{t \rightarrow \infty} x_{l}(t)=0, & \forall l \in\{r+1, \ldots, \rho\} .
\end{array}
$$

Remark 1: Such consensus objective covers different cases of interest including consensus in part of the states (exactly in $r$ of them) or in one output and its successive derivatives. For instance, for position-consensus of second-order mechanical systems we have $r=1$ and $\rho=2$. Hence, (7a) expresses the goal of achieving a desired consensual constant position modulo a desired offset for each system, (7b) is superfluous, and $(7 \mathrm{c})$ means that all velocities must converge to zero.

One of the advantages of considering the edge states rather than the node's is that it is possible to obtain an equivalent reduced system, easier to analyze using stability theory. Recall that, as observed in [27], using an appropriate labeling of the edges, the incidence matrix is expressed as

$$
E=\left[\begin{array}{ll}
E_{t} & E_{c}
\end{array}\right]
$$

where $E_{t} \in \mathbb{R}^{N \times(N-1)}$ denotes the full-column-rank incidence matrix corresponding to an arbitrary spanning tree $\mathcal{G}_{t} \subset \mathcal{G}$ and $E_{c} \in \mathbb{R}^{N \times(M-N+1)}$ represents the incidence matrix corresponding to the remaining edges not contained in $\mathcal{G}_{t}$. Moreover, defining

$$
R:=\left[\begin{array}{ll}
I_{N-1} & T
\end{array}\right]
$$

with $I_{N-1}$ denoting the $N-1$ identity matrix, and $T:=$ $\left(E_{t}^{\top} E_{t}\right)^{-1} E_{t}^{\top} E_{c}$, one obtains an alternative representation of the incidence matrix of the graph given by

$$
E=E_{t} R \text {. }
$$

The identity (10) is useful to derive a reduced-order dynamic model —cf. [27]. Such transformation is particularly useful to analyze consensus as a stabilization problem via Lyapunov's method [16]. Now, as in the latter references, the error edges' states, for each $l \in\{2, \ldots, r\}$, may be split as

$$
z_{l}=\left[\begin{array}{ll}
\tilde{z}_{l t}^{\top} & \tilde{z}_{l c}^{\top}
\end{array}\right]^{\top}, \quad \tilde{z}_{l t} \in \mathbb{R}^{n(N-1)}, \quad \tilde{z}_{l c} \in \mathbb{R}^{n(M-N+1)}
$$

where $z_{l t}$, for all $l \in\{1, \ldots, r\}$ are the states corresponding to the edges of an arbitrary spanning tree $\mathcal{G}_{t}$ and $\tilde{z}_{l c}$ denote the states of the remaining edges, $\mathcal{G} \backslash \mathcal{G}_{t}$. Thus, after (5), (6), and (11), denoting $z_{1 t}^{d} \in \mathbb{R}^{n(N-1)}$ as the vector of desired relative displacements corresponding to $\mathcal{G}_{t}$, we obtain

$$
\begin{aligned}
\tilde{z}_{1} & =\left[R^{\top} \otimes I_{n}\right] \tilde{z}_{1 t} \\
z_{l} & =\left[R^{\top} \otimes I_{n}\right] z_{l t}, \quad l \in\{2, \ldots, r\} .
\end{aligned}
$$

Now, collecting the inputs of the multiple agents into the vector $u^{\top}=\left[u_{1}^{\top} \cdots u_{N}^{\top}\right] \in \mathbb{R}^{n N}$ and the disturbances into $\theta_{l}^{\top}=\left[\theta_{1, l}^{\top} \cdots \theta_{N, l}^{\top}\right] \in \mathbb{R}^{n N}$, with $l \in\{1, \ldots, \rho\}$, the reduced-order system's equations read

$$
\begin{aligned}
\dot{\tilde{z}}_{1 t} & =z_{2 t}+\left[E_{t}^{\top} \otimes I_{n}\right] \theta_{1}(t) \\
\dot{z}_{l t} & =z_{(l+1) t}+\left[E_{t}^{\top} \otimes I_{n}\right] \theta_{l}(t), \quad l \in\{2, \ldots, r-1\} \\
\dot{z}_{r t} & =\left[E_{t}^{\top} \otimes I_{n}\right] x_{r+1}+\left[E_{t}^{\top} \otimes I_{n}\right] \theta_{r}(t) \\
\dot{x}_{l} & =x_{l+1}+\theta_{l}(t), \quad l \in\{r+1, \ldots, \rho-1\} \\
\dot{x}_{\rho} & =u+\theta_{\rho}(t) .
\end{aligned}
$$

In these coordinates, output-consensus as defined in (7) is achieved if the origin is asymptotically stabilized for the reduced-order system (14). More precisely we address the following problem.

Robust consensus problem with output constraints: Consider a multi-agent system of agents with high relative-degree dynamics given by (1), interacting either over a connected undirected graph, a directed spanning tree or a directed cycle. Assume, in addition, that the systems are subject to interagent constraints that consist in the outputs being restricted to remain in the set defined in (3). Under these conditions, find a distributed dynamic controller with outputs $u_{i}, i \leq N$, that, in the absence of disturbances, i.e., $\theta_{i, l} \equiv 0, l \leq \rho$, $i \leq N$, achieves the objective (7) and renders the constraints set (3) forward invariant, i.e., such that $z_{1}(0) \in \mathcal{D}$ imply that $z_{1}(t) \in \mathcal{D}$ for all $t \geq 0$. Furthermore, that in the presence of essentially bounded disturbances, that is $\theta_{l} \not \equiv 0, u_{i}$ render the origin of (14) practically input-to-state stable and the set $\mathcal{D}$ in (3) forward invariant. 


\section{CONTROL DESIGN FOR CONSENSUS UNDER OUTPUT CONSTRAINTS}

For clarity of exposition, we begin by presenting the control design for the output-consensus problem under output constraints. That is, in (7) let $r=1$, so these equations become

$$
\begin{aligned}
& \lim _{t \rightarrow \infty} \tilde{z}_{1}(t)=0 \\
& \lim _{t \rightarrow \infty} x_{l}(t)=0, \quad \forall l \in\{2, \ldots, \rho\} .
\end{aligned}
$$

More precisely, we consider the stabilization of the origin for

$$
\begin{aligned}
\dot{\tilde{z}}_{1 t} & =\left[E_{t}^{\top} \otimes I_{n}\right] x_{2}+\left[E_{t}^{\top} \otimes I_{n}\right] \theta_{1}(t) \\
\dot{x}_{l} & =x_{l+1}+\theta_{l}(t), \quad l \in\{2, \ldots, \rho-1\} \\
\dot{x}_{\rho} & =u+\theta_{\rho}(t) .
\end{aligned}
$$

Remark 2: In Section III-C farther below we show how the control methodology presented hereafter extends to cover the partial- and full-state consensus problem with output constraints defined in the previous section.

The control design for the system (16) follows a backstepping approach that naturally exploits the normal form of the system. Hence, we start by defining a virtual control law for (16a), using $x_{2}$ as input. Then, in order to account for the output constraints, a good choice of virtual input for (16a) consists in the gradient of a barrier Lyapunov function [36][38]. It is well known, however, that the backstepping approach may lead to an increase of complexity of the control law due to the successive differentiation of the virtual controllers. This problem is exacerbated by the fact that the said virtual control is designed as the gradient of a barrier function, which has multiple local minima and is defined only in open subsets of the state space. Therefore, in order to bypass these technical obstacles, inspired by the command filtered backstepping approach [26], we approximate the virtual inputs and their derivatives in each step of the backstepping design via command filters. This is explained in detail farther below.

\section{A. On barrier Lyapunov functions}

Barrier Lyapunov functions (BLFs) are reminiscent of Lyapunov functions, so they are positive definite, but their domain of definition is restricted by design to open subsets of the Euclidean space. Furthermore, they grow unbounded as the argument approaches the boundary of their domain. We define them as follows —cf. [24].

Definition 1 (Barrier Lyapunov function): Consider the system $\dot{x}=f(x)$ and let $\mathcal{J} \subset \mathbb{R}^{n}$ be an open set containing the origin. A Barrier Lyapunov function is a positive definite, function $V: \mathcal{J} \rightarrow \mathbb{R}_{\geq 0}, x \mapsto V(x)$, that is $\mathcal{C}^{1}$, satisfies

$$
\nabla V(x)^{\top} f(x):={\frac{\partial V(x)^{\top}}{\partial x}}^{\top} f(x) \leq 0,
$$

and has the property that $V(x) \rightarrow \infty$ and $|\nabla V(x)| \rightarrow \infty$ as $x \rightarrow \partial \mathcal{J}$.

Now, akin to (3), for each $k \leq M$, the inter-agent constraints in terms of the error coordinates are given by the set

$$
\tilde{\mathcal{D}}_{k}:=\left\{\tilde{z}_{k, 1} \in \mathbb{R}^{n}: \delta_{k}<\left|\tilde{z}_{k, 1}+z_{k, 1}^{d}\right|<\Delta_{k}\right\} .
$$

Then, for each $k \leq M$, we define a candidate BLF $W_{k}$ : $\tilde{\mathcal{D}}_{k} \rightarrow \mathbb{R}_{\geq 0}$, of the form

$$
W_{k}\left(\tilde{z}_{k, 1}\right)=\frac{1}{2}\left[\left|\tilde{z}_{k, 1}\right|^{2}+B_{k}\left(\tilde{z}_{k, 1}+z_{k, 1}^{d}\right)\right],
$$

where $B_{k}\left(\tilde{z}_{k, 1}+z_{k, 1}^{d}\right)$ is non-negative and satisfies: $B_{k}\left(z_{k, 1}^{d}\right)=0, \nabla B_{k}\left(z_{k, 1}^{d}\right)=0$, and $B_{k}\left(\tilde{z}_{k, 1}+z_{k, 1}^{d}\right) \rightarrow \infty$ as either $\left|\tilde{z}_{k, 1}+z_{k, 1}^{d}\right| \rightarrow \Delta_{k}$ or $\left|\tilde{z}_{k, 1}+z_{k, 1}^{d}\right| \rightarrow \delta_{k}$. Therefore, the candidate BLF (17) satisfies: $W_{k}\left(\tilde{z}_{k, 1}\right) \rightarrow \infty$ as either $\left|\tilde{z}_{k, 1}+z_{k, 1}^{d}\right| \rightarrow \Delta_{k}$ or $\left|\tilde{z}_{k, 1}+z_{k, 1}^{d}\right| \rightarrow \delta_{k}$, or equivalently in the original edge coordinates, as either $\left|z_{k, 1}\right| \rightarrow \Delta_{k}$ or $\left|z_{k, 1}\right| \rightarrow \delta_{k}$.

Remark 3: Note that $B_{k}\left(\tilde{z}_{k, 1}+z_{k, 1}^{d}\right)$ in (17) encodes the constraints on the original edge coordinates $z_{k, 1}$ in terms of the error $\tilde{z}_{k, 1}$. This may lead to imposing conservative feasibility conditions in terms of the initial conditions when using, e.g., logarithmic BLFs [39]. To overcome this limitation, $B_{k}\left(\tilde{z}_{k, 1}+\right.$ $\left.z_{k, 1}^{d}\right)$ may be defined as an integral BLF [39] or as a weight recentered barrier function [40] — see also Section V.

Remark 4: The functions defined in (17) are reminiscent of scalar potential functions in constrained environments [25] and, as for the latter, the appearance of multiple critical points is inevitable. Indeed, the gradient of the BLF (17), $\nabla W_{k}\left(\tilde{z}_{k, 1}\right)$, vanishes at the origin and at an isolated saddle point separated from the origin - see [41]. Therefore, when using the gradient of (17) for the control, the closed-loop system has multiple equilibria. This prevents us from using the classical stability tools for the analysis of the system. Such technical difficulty is addressed using tools tailored for so-called multi-stable systems—see [28], [29], and Appendix I.

\section{B. Control design for systems over directed graphs}

Let us define the so-called in-incidence matrix $E_{\odot} \in$ $\mathbb{R}^{N \times M}$, whose elements are defined as follows-cf. [42]$:\left[E_{\odot}\right]_{i k}:=-1$ if $i$ is the terminal node of edge $e_{k}$ and $\left[E_{\odot}\right]_{i k}:=0$ otherwise. Similarly the elements of so-called out-incidence matrix $E_{\otimes} \in \mathbb{R}^{N \times M}$ are defined as follows: $\left[E_{\odot}\right]_{i k}:=1$ if $i$ is the initial node of edge $e_{k}$ and $\left[E_{\odot}\right]_{i k}:=0$ otherwise. Define a BLF for the multi-agent system as

$$
W\left(\tilde{z}_{1}\right)=\sum_{k \leq M} W_{k}\left(\tilde{z}_{k, 1}\right),
$$

with $W_{k}\left(\tilde{z}_{k, 1}\right)$ given in (17) for all $k \leq M$, and denote by $\nabla W\left(\tilde{z}_{1}\right)$ the gradient of (18). Then, the virtual consensus control law for (16a), based on the gradient of the BLF $W$, is given by

$$
x_{2}^{*}:=-c_{1}\left[E_{\odot} \otimes I_{n}\right] \nabla W\left(\tilde{z}_{1}\right), \quad c_{1}>0 .
$$

Remark 5: For undirected graphs the virtual control law takes the form

$$
x_{2}^{*}=-c_{1}\left[E \otimes I_{n}\right] \nabla W\left(\tilde{z}_{1}\right), \quad c_{1}>0,
$$

where $E$ is the incidence matrix —cf. [38].

The right-hand side of (19) qualifies as a consensus control law that guarantees connectivity maintenance for first-order multi-agent systems $\dot{\tilde{z}}_{1 t}=u$ interconnected over directed 
graphs [16]. So, defining $\bar{x}_{2}:=x_{2}-x_{2}^{*}$ and using (19), Equation (16a) becomes ${ }^{1}$

$$
\dot{\tilde{z}}_{1 t}=-c_{1}\left[E_{t}^{\top} E_{\odot} \otimes I_{n}\right] \nabla W\left(\tilde{z}_{1}\right)+\left[E_{t}^{\top} \otimes I_{n}\right]\left[\bar{x}_{2}+\theta_{1}\right] .
$$

With aim at making $\bar{x}_{2} \rightarrow 0$ in (20), following a backstepping-based design, we rewrite the second equation in (16b), i.e., with $l=2$, in error coordinates $\bar{x}_{2}$ and we consider $x_{3}$ as an input. We have

$$
\dot{\bar{x}}_{2}=x_{3}-\dot{x}_{2}^{*}+\theta_{2} .
$$

Hence, the natural virtual control law at this stage is

$$
x_{3}^{*}=-c_{2} \bar{x}_{2}+\dot{x}_{2}^{*}, \quad c_{2}>0,
$$

which requires the derivative of the right-hand side of (19). Furthermore, a recursive procedure requires up to $\rho-2$ successive derivatives of $x_{2}^{*}$, which posses significant technical and numerical difficulties. Thus, to avoid the use of successive derivatives of $\nabla W\left(\tilde{z}_{1}\right)$ we approximate the derivatives of the virtual controls $x_{l}^{*}$, with $l \in\{2, \ldots, \rho-1\}$ by means of command filters. For simplicity, we use second-order systems defined as in the figure below —cf. [26];

$$
\stackrel{x_{l}^{*}}{\longrightarrow} H_{1}(s) \stackrel{x_{l f}}{\longrightarrow} \quad H_{1}(s):=\frac{\omega_{n}^{2}}{s^{2}+2 \omega_{n} s+\omega_{n}^{2}}
$$

Fig. 1: Command filter used for implementation. The dirty derivative of $x_{l}^{*}$ may be obtained using $\dot{x}_{l f}:=s H_{1}(s) x_{l}^{*}$ which is equivalent to $\dot{x}_{l f}=H_{1}(s) \dot{x}_{l}^{*}$.

The virtual controls are considered as the inputs of a command filter, with the outputs corresponding to the approximated signals and their derivatives, denoted $x_{l f}$ and $\dot{x}_{l f}$, respectively. The filters' natural frequency, $\omega_{n}>0$, is a control parameter which is chosen large enough so that the approximation $x_{l f}$ converges to the desired virtual control $x_{l}^{*}$ in a faster time-scale than that of the system's dynamics see Section IV-A. Moreover, the filters are designed with unit DC gain and unit damping coefficient so that the tracking of the virtual signals is guaranteed without overshoot. This ensures that, in the slower time-scale of the systems' dynamics, the "filtered forms" act as the desired virtual signals, corresponding to a classical backstepping control. Similarly, $\dot{x}_{l f}=H_{1}(s) \dot{x}_{l}^{*}$ approximates $\dot{x}_{l}^{*}$.

Remark 6: For clarity, we use second-order commandfilters as defined in Fig. 1 above. However, the design is not restricted to this particular choice. Indeed, other possibilities include first-order low-pass filters [43] or high-order Levant differentiators [34].

For the purpose of stability analysis, we write the command filters' dynamics in state form. To that end, let the filters' variables be denoted as $\alpha_{l-1}^{\top}:=\left[\begin{array}{ll}\alpha_{l-1,1}^{\top} & \alpha_{l-1,2}^{\top}\end{array}\right] \in \mathbb{R}^{2 n N}$, for $l \in\{2, \ldots, \rho\}$. Then, in state-space representation, the command filters are written as

$$
\dot{\alpha}_{l-1}=\omega_{n}\left[A \otimes I_{n N}\right] \alpha_{l-1}+\omega_{n}\left[B \otimes I_{n N}\right] x_{l}^{*}
$$

\footnotetext{
${ }^{1}$ To avoid a cumbersome notation we write $\nabla W\left(\tilde{z}_{1}\right)$ in place of the more appropriate spelling $\nabla W\left(\left[R^{\top} \otimes I_{n}\right] \tilde{z}_{1 t}\right)$.
}

$$
\begin{aligned}
& {\left[x_{l f}^{\top} \dot{x}_{l f}^{\top}\right]^{\top}=\left[C \otimes I_{n N}\right] \alpha_{l-1}, \quad l \in\{2, \ldots, \rho\}} \\
& A:=\left[\begin{array}{cc}
0 & 1 \\
-1 & -2
\end{array}\right], \quad B:=\left[\begin{array}{l}
0 \\
1
\end{array}\right], \quad C:=\left[\begin{array}{cc}
1 & 0 \\
0 & \omega_{n}
\end{array}\right]
\end{aligned}
$$

and the initial conditions are set to $\alpha_{l-1,1}(0)=x_{l}^{*}(0)$ and $\alpha_{l-1,2}(0)=0$.

Thus, the virtual control inputs, starting with (22), are redefined using the filter variables as follows. First, we redefine

$$
x_{3}^{*}:=-c_{2} \tilde{x}_{2}+\dot{x}_{2 f}
$$

where $\tilde{x}_{2}:=x_{2}-x_{2 f}$ and $\dot{x}_{2 f}=\omega_{n} \alpha_{1,2}$. Hence, in contrast to $(21)$, from

$$
\dot{x}_{2}-\dot{x}_{2 f}=x_{3}-\dot{x}_{2 f}+\theta_{2}+x_{3}^{*}-x_{3}^{*}+\alpha_{2,1}-\alpha_{2,1},
$$

defining $\tilde{x}_{3}:=x_{3}-x_{3 f}$ and using (24) and $\alpha_{2,1}=x_{3 f}$, we obtain

$$
\dot{\tilde{x}}_{2}=-c_{2} \tilde{x}_{2}+\tilde{x}_{3}+\left(\alpha_{2,1}-x_{3}^{*}\right)+\theta_{2} .
$$

Then, owing to the fact that the system is in feedback form, we define

$$
x_{l}^{*}:=-c_{l-1} \tilde{x}_{l-1}+\omega_{n} \alpha_{l-2,2}-\tilde{x}_{l-2}, \quad l \in\{4, \ldots, \rho\},
$$

- cf. Eq. (24), where $c_{2}, c_{l-1}$ are positive constants, and the tracking errors are given by

$$
\tilde{x}_{l}:=x_{l}-x_{l f}=x_{l}-\alpha_{l-1,1}, \quad l \in\{2, \ldots, \rho\} .
$$

That is, the virtual controls $x_{l}^{*}$ starting from $l=3$ are redesigned to steer $x_{l-1}$ towards the filtered virtual input $x_{l-1 f}$. Finally, the actual control input is set to

$$
u=-c_{\rho} \tilde{x}_{\rho}+\omega_{n} \alpha_{\rho-2,2}-\tilde{x}_{\rho-1} .
$$

Remark 7: The system being in feedback form, the third term on the right-hand side of (25) and (27) are feedback passivation terms — cf. [44]. These terms, that come from the backstepping-as-recursive-feedback-passivation approach [45] are used to render the system (14) passive with respect to the output $y_{\rho}:=x_{\rho}-x_{\rho f}$.

Thus, taking the derivative of the backstepping error variables defined in (26) and using the input (27), with (23)-(25), we obtain the closed-loop system

$$
\begin{aligned}
& \dot{\tilde{z}}_{1 t}=- c_{1}\left[E_{t}^{\top} E_{\odot} \otimes I_{n}\right] \nabla W\left(\tilde{z}_{1}\right) \\
&+\left[E_{t}^{\top} \otimes I_{n}\right]\left[\tilde{x}_{2}+\left(\alpha_{1,1}-x_{2}^{*}\right)+\theta_{1}\right] \\
& \dot{\tilde{x}}_{2}=-c_{2} \tilde{x}_{2}+\tilde{x}_{3}+\left(\alpha_{2,1}-x_{3}^{*}\right)+\theta_{2} \\
& \dot{\tilde{x}}_{l}=-c_{l} \tilde{x}_{l}+\tilde{x}_{l+1}-\tilde{x}_{l-1}+\left(\alpha_{l, 1}-x_{l+1}^{*}\right)+\theta_{l}, \\
& \forall l \in\{3, \ldots, \rho-1\} \\
& \dot{\tilde{x}}_{\rho}=-c_{\rho} \tilde{x}_{\rho}-\tilde{x}_{\rho-1}+\theta_{\rho} \quad \\
& \dot{\alpha}_{l}=\omega_{n}\left[A \otimes I_{n N}\right] \alpha_{l}+\omega_{n}\left[B \otimes I_{n N}\right] x_{l+1}^{*}, \\
& \forall l \in\{1, \ldots, \rho-1\} .
\end{aligned}
$$

Consequently, solving the robust consensus problem with output constraints comes to guaranteeing that $\tilde{z}_{1 t}(t)$, as part of the solution to Eqs. (28), tends to zero. More precisely, that the control law (27), with (19) and (23)-(25), solves the robust output-consensus problem with output constraints for system (1) is a fact established by the following statement. 
Theorem 1: Consider the system (1) in closed loop with the dynamic controller defined by (27), together with (19), (24)-(25), and the command filters (23) with initial conditions set to $\alpha_{l-1,1}(0)=x_{l}^{*}(0)$ and $\alpha_{l-1,2}(0)=0, l \in\{2, \ldots, \rho\}$. Then, under the Standing Assumption, the constraints set (3) is forward invariant. Moreover, if $\theta_{i, l} \equiv 0, l \leq \rho, i \leq N$, (15) holds for almost all initial conditions satisfying $z_{1}(0) \in \mathcal{D}$. Otherwise, if $\theta_{l} \not \equiv 0$, the closed-loop system is almosteverywhere practically input-to-state stable with respect to $\theta:=\left[\theta_{1}^{\top} \cdots \theta_{\rho}^{\top}\right]^{\top}$.

For clarity of exposition, the proof of Theorem 1 is presented in Section IV, but in anticipation of the latter we underline the following. The closed-loop system (28) may be separated into two systems evolving in distinct time scales. One fast system that corresponds to the command filters equations (28e) and one slow system that corresponds to the actual system under ordinary backstepping control -Eqs. (28a)-(28d). Therefore, choosing the fundamental frequency $\omega_{n}$ relatively large and multiplying by $\epsilon:=1 / \omega_{n}$ on both sides of (28e) we see that the system (28) is in singular-perturbation form [46] and may be analyzed as such. The reduced slow system may be analyzed using Lyapunov and input-to-state stability theory, but because $W$ has multiple equilibria, we use a refinement of these theories, in a multi-stability sense [28], [29]. This is significant to establish not only convergence to the consensus manifold but also explicit robustness properties in the sense of practical input-to-state stability with respect to bounded disturbances.

\section{Extension to partial- and full-state consensus with output constraints}

As mentioned previously, the control design methodology presented above for output consensus can be directly extended to consider the objective (7) with any $r>1$. To show this, let

$$
\begin{aligned}
& \tilde{z}_{l}:=z_{l}-\left[E^{\top} \otimes I_{n}\right] x_{l f} \quad \forall l \in\{2, \ldots, r\} \\
& \tilde{x}_{l}:=x_{l}-x_{l f} \quad \forall l \in\{r+1, \ldots, \rho\},
\end{aligned}
$$

where, we recall, $x_{l f}=\alpha_{l-1,1}$. Then, to achieve the consensus objectives (7) the virtual control inputs, at each step, are redefined as

$$
z_{l}^{*}:=\left[E^{\top} \otimes I_{n}\right] x_{l}^{*}, \quad \forall l \in\{2, \ldots, r\}
$$

with $x_{2}^{*}$ as in (19),

$$
\begin{aligned}
& x_{3}^{*}:=-c_{2}\left[E_{\odot} \otimes I_{n}\right] \tilde{z}_{2}+\omega_{n} \alpha_{1,2} \\
& x_{l}^{*}:=-c_{l-1}\left[E_{\odot} \otimes I_{n}\right] \tilde{z}_{l-1}+\omega_{n} \alpha_{l-2,2}-\tilde{x}_{l-2}
\end{aligned}
$$

for all $l \in\{4, \ldots, r\}$, and

$$
\begin{gathered}
x_{r+1}^{*}:=-c_{r}\left[E_{\odot} \otimes I_{n}\right] \tilde{z}_{r}+\omega_{n} \alpha_{r-1,2}-\left[E_{\odot} \otimes I_{n}\right] \tilde{z}_{r-1}, \\
x_{l}^{*}:=-c_{l-1} \tilde{x}_{l-1}+\omega_{n} \alpha_{l-2,2}-\tilde{x}_{l-2}, \\
\forall l \in\{r+2, \ldots, \rho\} .
\end{gathered}
$$

Finally, the actual control input is set to

$$
u:=-c_{\rho} \tilde{x}_{\rho}+\omega_{n} \alpha_{\rho-2,2}-\tilde{x}_{\rho-1} .
$$

Then, we have the following.
Theorem 2: Consider the system (1) in closed loop with the dynamic controller defined by (36), together with (31)(35), and the command filters (23) with initial conditions set to $\alpha_{l-1,1}(0)=x_{l}^{*}(0)$ and $\alpha_{l-1,2}(0)=0, l \in\{2, \ldots, \rho\}$. Then, under the Standing Assumption the constraints set (3) is forward invariant. Moreover, if $\theta_{i, l} \equiv 0, l \leq \rho, i \leq$ $N$, the limits in (7) hold for almost all initial conditions satisfying $z_{1}(0) \in \mathcal{D}$. Otherwise, if $\theta_{l} \not \equiv 0$, the closed-loop system is almost-everywhere practically input-to-state stable with respect to $\theta:=\left[\theta_{1}^{\top} \cdots \theta_{\rho}^{\top}\right]^{\top}$.

Note that the virtual inputs (32)-(34) may be considered proportional to the gradient of a quadratic function on $\tilde{z}_{l}$, respectively, for $l \in\{2, \ldots, r\}$. Hence, modulo the stability analysis of the additional edge coordinates $\tilde{z}_{l}$, for $l \in$ $\{2, \ldots, r\}$, which is similar to that of the output consensus error $\tilde{z}_{1}$, the proof is identical to the proof of Theorem 1 .

\section{PROOF OF THEOREM 1}

The proof of the statement for arbitrary undirected graphs is strictly contained within that for directed graphs. The latter is organized in two main parts. First, we show how the closedloop system (28) can be written as a singularly-perturbed system in which the fast systems correspond to the dynamics of the command filters and the slow system corresponds to the high-order dynamics of the original multi-agent system. Then, we analyze the stability and the robustness of the singularly perturbed system, using multi-stable systems theory [28], [29].

\section{A. Singular-perturbation representation}

Define $\alpha^{\top}:=\left[\alpha_{1}^{\top} \cdots \alpha_{\rho-1}^{\top}\right] \in \mathbb{R}^{2 n N(\rho-1)}, \xi^{\top}:=$ $\left[\tilde{z}_{1 t}^{\top} \tilde{x}_{2}^{\top} \cdots \tilde{x}_{\rho}\right]^{\top} \in \mathbb{R}^{n(\rho N-1)}$, and $\theta^{\top}:=\left[\theta_{1} \cdots \theta_{\rho}\right] \in$ $\mathbb{R}^{n \rho N}$. Then, the filter subsystem (28e) can be rewritten as

$$
\begin{gathered}
\left.\left.\dot{\alpha}=\omega_{n} \tilde{A}[\alpha-\chi(\xi, \alpha)], \quad \begin{array}{l}
\tilde{A}:=\operatorname{blockdiag}\left\{\left[A \otimes I_{n N}\right.\right.
\end{array}\right]\right\}, \\
\chi(\xi, \alpha):=\left[\begin{array}{lllllll}
x_{2}^{* \top} & 0^{\top} & x_{3}^{* \top} & 0^{\top} & \cdots & x_{\rho}^{* \top} & 0^{\top}
\end{array}\right]^{\top} .
\end{gathered}
$$

Now, as mentioned in the previous section, with $\epsilon:=1 / \omega_{n}$ as the singular parameter, the closed-loop system (28) may be written in the singular-perturbation form

$$
\begin{aligned}
\dot{\xi} & =f(\xi, \alpha, \theta, \epsilon) \\
\epsilon \dot{\alpha} & =g(\xi, \alpha, \theta, \epsilon)
\end{aligned}
$$

and setting $\epsilon=0$ in (38) we obtain the quasi-state model [46],

$$
\begin{aligned}
\dot{\xi} & =f(\xi, \alpha, \theta, 0) \\
0 & =g(\xi, \alpha, \theta, 0)
\end{aligned}
$$

in which (39b) becomes an algebraic equation. Hence, the analysis of the singular perturbation model (38) is normally conducted studying its dynamic properties in different time scales.

Denote by $\alpha_{s}=h(\xi)$ the unique root of the algebraic equation (39b), i.e.,

$$
\begin{aligned}
h(\xi)=\left[\left[-c_{1}\left[E_{\odot} \otimes I_{n}\right] \nabla W\left(\tilde{z}_{1}\right)\right]^{\top}\right. & 0^{\top}-c_{2} \tilde{x}_{2}^{\top} 0^{\top} \\
\cdots & \left.-c_{\rho-1} \tilde{x}_{\rho-1}^{\top} 0^{\top}\right]^{\top} .
\end{aligned}
$$


Then, defining the coordinate transformation

$$
\tilde{\alpha}:=\left[\begin{array}{lllll}
\tilde{\alpha}_{1,1}^{\top} & \tilde{\alpha}_{1,2}^{\top} & \cdots & \tilde{\alpha}_{\rho-1,1}^{\top} & \tilde{\alpha}_{\rho-1,2}^{\top}
\end{array}\right]^{\top}=\alpha-h(\xi),
$$

and using (28) we obtain the singularly-perturbed system

$$
\begin{aligned}
& \dot{\tilde{z}}_{1 t}=- c_{1}\left[E_{t}^{\top} E_{\odot} \otimes I_{n}\right] \nabla W\left(\tilde{z}_{1}\right) \\
&+\left[E_{t}^{\top} \otimes I_{n}\right]\left[\tilde{x}_{2}+\tilde{\alpha}_{1,1}+\theta_{1}\right] \\
& \dot{\tilde{x}}_{2}=- c_{2} \tilde{x}_{2}+\tilde{x}_{3}+\tilde{\alpha}_{2,1}+\theta_{2} \\
& \dot{\tilde{x}}_{l}=-c_{l} \tilde{x}_{l}+\tilde{x}_{l+1}-\tilde{x}_{l-1}+\tilde{\alpha}_{l, 1}+\theta_{l}, \\
& \quad l \in\{3, \ldots, \rho-1\} \\
& \dot{\tilde{x}}_{\rho}=-c_{\rho} \tilde{x}_{\rho}-\tilde{x}_{\rho-1}+\theta_{\rho} \\
& \epsilon \dot{\tilde{\alpha}}=\tilde{A} \tilde{\alpha}-\epsilon \frac{\partial h(\xi)}{\partial \xi} \dot{\xi} .
\end{aligned}
$$

In turn, the reduced system $\dot{\xi}=f(\xi, h(\xi), \theta, 0)$ takes the form

$$
\begin{aligned}
\dot{\tilde{z}}_{1 t} & =-c_{1}\left[E_{t}^{\top} E_{\odot} \otimes I_{n}\right] \nabla W\left(\tilde{z}_{1}\right)+\left[E_{t}^{\top} \otimes I_{n}\right]\left[\tilde{x}_{2}+\theta_{1}\right] \\
\dot{\bar{x}} & =-\left[H \otimes I_{n N}\right] \bar{x}+\bar{\theta}
\end{aligned}
$$

where $\bar{x}^{\top}:=\left[\begin{array}{lll}\tilde{x}_{2}^{\top} & \cdots & \tilde{x}_{\rho}^{\top}\end{array}\right], \bar{\theta}^{\top}:=\left[\begin{array}{lll}\theta_{2}^{\top} & \cdots & \theta_{\rho}^{\top}\end{array}\right]$, and

$$
H:=\left[\begin{array}{ccccc}
c_{2} & -1 & 0 & \ldots & 0 \\
1 & c_{3} & -1 & \ldots & 0 \\
\vdots & \ddots & \ddots & \ddots & \vdots \\
0 & \ldots & 1 & c_{\rho-1} & -1 \\
0 & \ldots & 0 & 1 & c_{\rho}
\end{array}\right]
$$

On the other hand, the boundary layer system, $(d \tilde{\alpha} / d \tau)=$ $g(\xi, \tilde{\alpha}+h(\xi), \theta, 0)$, with $\tau=t / \epsilon$ and with $\xi$ considered as fixed, is

$$
\frac{d \tilde{\alpha}}{d \tau}=\tilde{A} \tilde{\alpha}
$$

where $\tilde{A}$ is a Hurwitz matrix - see Eqs. (37) and (23c).

Note that even though the system (41) appears to be in the familiar form (38), the analysis of the slow dynamics (41a)(41d) subject to $\epsilon=0$ (hence to $\tilde{\alpha}=0$ ), that is, Eqs. (42), is stymied by the fact that the function $\nabla W$ vanishes at multiple separate equilibria. Therefore, we use perturbation theory for multi-stable systems [28] to establish input-to-state stability. The analysis is provided below and some definitions and statements from the latter reference are recalled in Appendix I, for convenience.

\section{B. Stability and robustness analysis}

Denote $\tilde{z}_{1 t}^{*} \in \mathbb{R}^{n(N-1)}$ as the vector containing the saddle points of the BLF for each edge (17). Then, the equilibrium points of subsystem (41a) are collected into a disjoint set, denoted by

$$
\mathcal{W}:=\{0\} \cup\left\{\tilde{z}_{1 t}^{*}\right\},
$$

which is an acyclic $\mathcal{W}$-limit $\operatorname{set}^{2}$ of (41a). This means that asymptotic stability of the origin of (41a) may be guaranteed, at best, almost everywhere in $\mathcal{D}$, that is, for all initial conditions in $\mathcal{D}$ except for a set of measure zero corresponding to the domain of attraction of the unstable critical point.

\footnotetext{
${ }^{2}$ In the settings of this paper an acyclic limit set corresponds to an invariant set of isolated points in Euclidean space. See [28] for a complete definition.
}

Now, we first analyze the stability of (41) with respect to the set of equilibria $\mathcal{W}_{\Theta}:=\mathcal{W} \times\{0\}^{\rho-1}$. For this purpose we use Theorem 3 in Appendix I, which is essentially a reformulation of [28, Theorem 2] adapted to the contents of this paper. Theorem 3 establishes sufficient conditions for a practical input-to-state multi-stability property to hold for a singularly perturbed system with respect to $\mathcal{W}_{\Theta}$ and to a bounded external input $\theta$, granted that the reduced system (42) is input-to-state stable with respect to set $\mathcal{W}_{\Theta}$ and input $\theta$ and that the origin for (44) is globally asymptotically stable. Therefore, the stability and robustness analysis is conducted in the following steps:

1) We show that the origin is asymptotically stable for the boundary layer system (44).

2) Relying on the results on cascaded multi-stable systems in [29], we show that the reduced system (42) is inputto-state stable with respect to set $\mathcal{W}_{\Theta}$ and input $\theta$.

3) Using Theorem 3 in Appendix I, we prove that, for a sufficiently small $\epsilon$, the singularly perturbed system (41) is practically input-to-state stable with respect to the set $\mathcal{W}_{\Theta} \times\{0\}$ and a bounded external input $\theta$. Moreover, using [28, Theorem 3], in the absence of disturbances, we show convergence to the set of equilibria.

4) Using the practical input-to-state multi-stability property, we establish almost-everywhere-practical-input-tostate stability of the origin of (41). Similarly, if $\theta \equiv 0$, we establish convergence to the origin.

5) Finally, we show that the output-constraints set defined in (3) is forward invariant.

Step 1) Since $\tilde{A}$ is Hurwitz by design, the origin $\tilde{\alpha}=0$ is exponentially stable for the boundary-layer system (44).

Step 2) Consider the reduced system (42). Note that it has the form of a cascaded system, in which (42b) is the "driving" system and the "driven" system (42a) has multiple equilibria given by the set $\mathcal{W}$ in (45). In order to prove input-to-state stability of (42) with respect to set $\mathcal{W}_{\Theta}$ and input $\theta$, as per in [29], we need to show that (42a) is input-to-state stable with respect to the set $\mathcal{W}$ and the inputs $\tilde{x}_{2}$ and $\theta_{1}$ whereas the system (42b) is input-to-state stable with respect to $\bar{\theta}$. We start with the latter.

Input-to-state stability with respect to $\bar{\theta}$ for the system (42b) follows directly from Lyapunov theory since (42b) is a linear time-invariant system and $-\left[H \otimes I_{n N}\right]$ is Hurwitz matrix, since so is $-H$.

Consider, in turn, the reduced subsystem (42a). For this system we use the BLF $W\left(\tilde{z}_{1}\right)$ given by (18). First note that $W\left(\tilde{z}_{1}\right)$ consists in a sum of the BLFs defined for each edge in the initial graph. Yet, from the identity (12) it is possible to express $W$ in terms of the edges corresponding to a spanning tree contained in the graph. Hence, define the candidate Lyapunov function

$$
V_{z}\left(\tilde{z}_{1 t}\right)=W\left(\left[R^{\top} \otimes I_{n}\right] \tilde{z}_{1 t}\right) .
$$

On the other hand, for consistency in the notation, we define the constraint set (3) in terms of the edges of the spanning tree $z_{1 t}$ as

$$
\mathcal{D}_{t}:=\left\{z_{1 t} \in \mathbb{R}^{n(N-1)}:\left|\bar{r}_{k}^{\top} z_{k, 1}\right| \in\left(\delta_{k}, \Delta_{k}\right), \forall k \leq M\right\},
$$


where $\bar{r}_{k}:=\left[r_{k} \otimes I_{n}\right]$ and $r_{k}$ is the $k$ th column of $R$ in (9). For the time-being, assume that $\mathcal{D}$ (equivalently $\mathcal{D}_{t}$ ) is forward invariant; this hypothesis is relaxed below. Then, for all $\tilde{z}_{1 t}$ such that $z_{1 t} \in \mathcal{D}_{t}, V_{z}\left(\tilde{z}_{1 t}\right)$ satisfies

$$
\frac{1}{2}\left|\tilde{z}_{1 t}\right|_{\mathcal{W}}^{2} \leq V_{z}\left(\tilde{z}_{1 t}\right)
$$

where $\left|\tilde{z}_{1 t}\right| \mathcal{W}=\min \left\{\left|\tilde{z}_{1 t}\right|,\left|\tilde{z}_{1 t}-\tilde{z}_{1 t}^{*}\right|\right\}$. Furthermore, from (42a), the derivative of $V_{z}$ is given by

$$
\begin{aligned}
\dot{V}_{z}= & -c_{1} \nabla W\left(\tilde{z}_{1}\right)\left[R^{\top} E_{t}^{\top} E_{\odot} \otimes I_{n}\right] \nabla W\left(\tilde{z}_{1}\right) \\
& +\nabla W\left(\tilde{z}_{1}\right)^{\top}\left[R^{\top} E_{t}^{\top} \otimes I_{n}\right]\left[\tilde{x}_{2}+\theta_{1}\right],
\end{aligned}
$$

where $R$ is defined in (9). Equation (47) holds for, both, directed-spanning-tree and directed-cycle topologies. Next, we analyze the two considered topologies separately.

Case 1 (Directed spanning tree). In this case we have $\mathcal{G}=$ $\mathcal{G}_{t}$. Therefore, $z_{1}=z_{1 t}, E=E_{t}, R=I_{N-1}$, and $E_{\odot}=E_{\odot t}$, where $E_{\odot t}$ is the in-incidence matrix of the spanning tree. Hence, (47) becomes

$$
\begin{aligned}
\dot{V}_{z}= & -c_{1} \nabla W\left(\tilde{z}_{1 t}\right)\left[E_{t}^{\top} E_{\odot t} \otimes I_{n}\right] \nabla W\left(\tilde{z}_{1 t}\right) \\
& +\nabla W\left(\tilde{z}_{1 t}\right)^{\top}\left[E_{t}^{\top} \otimes I_{n}\right]\left[\tilde{x}_{2}+\theta_{1}\right] .
\end{aligned}
$$

Define $L_{e}^{s}:=\frac{1}{2}\left(E_{t}^{\top} E_{\odot t}+E_{\odot t}^{\top} E_{t}\right)$, which is the symmetric part of the so-called directed edge Laplacian $E_{t}^{\top} E_{\odot t}$. As it is shown in the proof of Proposition 1 in [16], $L_{e}^{s}$ is positive definite. Therefore, applying Young's inequality to the second term in the right-hand side of (48), we have

$$
\dot{V}_{z} \leq-c_{1}^{\prime}\left|\nabla V_{z}\left(\tilde{z}_{1 t}\right)\right|^{2}+\frac{1}{2 \gamma}\left[\left|\tilde{x}_{2}\right|^{2}+\left|\theta_{1}\right|^{2}\right],
$$

where $c_{1}^{\prime}:=\left[c_{1} \lambda_{\min }\left(L_{e}^{s}\right)-\frac{\gamma \lambda_{\max }\left(E_{t}^{\top} E_{t}\right)}{2}\right]$ is positive for a sufficiently small $\gamma>0, \lambda_{\min }\left(L_{e}^{s}\right)>0$ is the smallest eigenvalue of $L_{e}^{s}$, and $\lambda_{\max }\left(E_{t}^{\top} E_{t}\right)$ is the largest eigenvalue of $E_{t}^{\top} E_{t}$.

Case 2 (Directed cycle). Consider equation (47). Using the identity (10), we have

$$
\begin{aligned}
\dot{V}_{z}= & -c_{1} \nabla W\left(\tilde{z}_{1}\right)\left[E^{\top} E_{\odot} \otimes I_{n}\right] \nabla W\left(\tilde{z}_{1}\right) \\
& +\nabla W\left(\tilde{z}_{1}\right)^{\top}\left[E^{\top} \otimes I_{n}\right]\left[\tilde{x}_{2}+\theta_{1}\right] .
\end{aligned}
$$

Now, for a directed-cycle topology, the following identity follows -cf. [16]-

$$
E^{\top} E_{\odot}+E_{\odot}^{\top} E=E^{\top} E .
$$

Therefore, using (51) and (10) again, we obtain

$$
\begin{aligned}
\dot{V}_{z}= & -\frac{c_{1}}{2} \nabla W\left(\tilde{z}_{1}\right)\left[R^{\top} E_{t}^{\top} E_{t} R^{\top} \otimes I_{n}\right] \nabla W\left(\tilde{z}_{1}\right) \\
& +\nabla W\left(\tilde{z}_{1}\right)^{\top}\left[R^{\top} E_{t}^{\top} \otimes I_{n}\right]\left[\tilde{x}_{2}+\theta_{1}\right] .
\end{aligned}
$$

Denote

$$
\nabla V_{z}\left(\tilde{z}_{1 t}\right):=\frac{\partial V_{z}\left(\tilde{z}_{1 t}\right)}{\partial \tilde{z}_{1 t}}=\left[R \otimes I_{n}\right] \nabla W\left(\left[R^{\top} \otimes I_{n}\right] \tilde{z}_{1 t}\right) .
$$

Then, applying Young's inequality to the second term of the right-hand side of (52), we have

$$
\dot{V}_{z} \leq-c_{1}^{\prime}\left|\nabla V_{z}\left(\tilde{z}_{1 t}\right)\right|^{2}+\frac{1}{2 \gamma}\left[\left|\tilde{x}_{2}\right|^{2}+\left|\theta_{1}\right|^{2}\right],
$$

where $c_{1}^{\prime}:=\frac{\lambda_{\min }\left(E_{t}^{\top} E_{t}\right)}{2}\left[c_{1}-\gamma\right]$ is positive for a sufficiently small $\gamma>0$. Note that, $\lambda_{\min }\left(E_{t}^{\top} E_{t}\right)$ is positive since it is the smallest eigenvalue of the edge Laplacian of an undirected spanning tree - see [38].

Thus, for either the spanning tree or the cycle case, the derivative of $V_{z}\left(z_{1 t}\right)$ satisfies

$$
\dot{V}_{z} \leq-c_{1}^{\prime}\left|\tilde{z}_{1 t}\right|_{\mathcal{W}}^{2}+\frac{1}{2 \gamma}\left[\left|\tilde{x}_{2}\right|^{2}+\left|\theta_{1}\right|^{2}\right] .
$$

It follows from (46), (55), and Theorem 4 in Appendix I, that the subsystem (42a) is input-to-state stable with respect to the set of equilibria $\mathcal{W}$, and the inputs $\tilde{x}_{2}$ and $\theta_{1}$.

Thus, after [29, Theorem 3.1], the reduced system (42) is input-to-state stable with respect to $\mathcal{W}_{\Theta}$ and input $\theta$. Furthermore, $\mathcal{W}_{\Theta}$ qualifies as a $\mathcal{W}$-limit set for (42).

Remark 8: In the case of an interaction topology given by an arbitrary connected undirected graph, it is shown in [38] that the input-to-state stability result (55) follows from (47) (replacing $E_{\odot}$ by $E_{t} R$ ) by noting that $E_{t}^{\top} E_{t}$ is symmetric positive definite [27], to obtain directly (52).

Remark 9: Note that for generic connected directed graphs the symmetric part of the directed edge Laplacian $E^{\top} E_{\odot}$ is not positive semi-definite. Indeed, in such cases, the identity (51) does not hold. Moreover, for the in-incidence matrix of a general directed graph there does not exist an equivalent identity to (10). Hence, unlike for connected undirected graphs - see, e.g., [38], the directed edge Laplacian of a general directed graph cannot be transformed into the directed edge Laplacian of a spanning tree $E_{t}^{\top} E_{\odot t}$, which has a positivedefinite symmetric part. Thus, the analysis of the constrainedconsensus problem in edge coordinates over general directed graphs, via strict and ISS Lyapunov functions, is still an open problem.

Step 3) Since the reduced system (42) is input-to-state stable with respect to $\mathcal{W}_{\Theta}$ and $\theta$, and the origin of (44) is asymptotically stable, it follows, after Theorem 3 in Appendix I, that the singularly perturbed system (41) is practically input-tostate stable with respect to set $\mathcal{W}_{\Theta} \times\{0\}^{2 n N(\rho-1)}$ and input $\theta$. More precisely, for any pair of constants $d_{1}, d_{2}>0$, there exists an $\epsilon^{*}>0$ such that, for any $\epsilon \in\left(0, \epsilon^{*}\right]$, the solutions of (41) satisfy

$$
\begin{aligned}
\limsup _{t \rightarrow \infty}|\xi(t)| \mathcal{W}_{\Theta} & \leq \eta_{\theta}\left(\|\theta\|_{\infty}\right)+d_{2} \\
|\tilde{\alpha}(t)| & \leq \beta_{\alpha}\left(|\tilde{\alpha}(0)|, \frac{t}{\epsilon}\right)+d_{2}, \quad \forall t \geq 0,
\end{aligned}
$$

provided that

$$
\max \left\{|\xi(0)|_{\mathcal{W}_{\Theta}},|\tilde{\alpha}(0)|,\|\theta\|_{\infty},\|\dot{\theta}\|_{\infty}\right\} \leq d_{1}
$$

where $\|\theta\|_{\infty}:=\limsup _{t \rightarrow \infty}|\theta(t)|$ and $|\xi|_{\mathcal{W}_{\Theta}}:=\inf _{a \in \mathcal{W}_{\Theta}}|\xi-a|$.

Now consider the case in which the disturbance $\theta \equiv 0$. From property (56a) we may conclude that the origin of the reduced system (42) is multi-stable with respect to $\mathcal{W}_{\Theta}$. Therefore, from the latter and the exponential stability of the boundary layer system (44) all the assumptions of [28, Theorem 3] are satisfied and the solutions of (41) satisfy

$$
\lim _{t \rightarrow \infty}|\xi(t)|_{\mathcal{W}_{\ominus}}=0
$$




$$
\lim _{t \rightarrow \infty}|\tilde{\alpha}(t)|=0 \text {. }
$$

Step 4) Since the critical point $\tilde{z}_{1 t}^{*}$ of the BLF is a saddle point - see [41], after [47, Proposition 11], it follows that the region of attraction of the unstable equilibrium $\tilde{z}_{1 t}^{*}$ has zero Lebesgue measure. Therefore, we conclude that the bound in (56a) and the limit in (57a) are satisfied for the origin $\{\xi=0\}$. More precisely, we have

$$
\limsup _{t \rightarrow \infty}|\xi(t)| \leq \eta_{\theta}\left(\|\theta\|_{\infty}\right)+d_{2}
$$

and, for $\theta \equiv 0$,

$$
\lim _{t \rightarrow \infty}|\xi(t)|=0
$$

Step 5) Up to this point we have assumed that the interagent constraints are satisfied for all time, that is, $z_{1}(t) \in \mathcal{D}$ for all $t \geq 0$. Then, in order to prove the forward invariance of the constraints set we proceed by contradiction as follows. Assume that the state constraints are not respected. Therefore, from continuity of the solutions, there exists a time $T>0$ such that $z_{1 t}(T) \in \partial \mathcal{D}_{t}$. Now, from the previous analysis of the singularly perturbed system, we have that in the interval $[0, T)$, condition (83) holds. Moreover, since $\tilde{\alpha}(0)=0$ by design, the solutions of the filter error satisfy $\tilde{\alpha}(t) \leq d_{2}$, for $t \in[0, T)$. Consider the derivative of the Lyapunov function (46) along the trajectories of (41a), which satisfies

$$
\dot{V}_{z}\left(\tilde{z}_{1 t}\right) \leq-c_{1}^{\prime}\left|\nabla V_{z}\left(\tilde{z}_{1 t}\right)\right|^{2}+\frac{1}{2 \gamma}\left[\left|\tilde{x}_{2}\right|^{2}+\left|\theta_{1}\right|^{2}+|\tilde{\alpha}|^{2}\right] .
$$

Therefore, since $\tilde{\alpha}(t) \leq d_{2}, \theta_{1}(t)$ is bounded and the system in (42b) is input-to-state stable, $\tilde{x}_{2}(t)$ is bounded, for all $t \in$ $[0, T)$. Then, we have

$$
\dot{V}_{z}\left(\tilde{z}_{1 t}(t)\right) \leq-c_{1}^{\prime}\left|\nabla V_{z}\left(\tilde{z}_{1 t}(t)\right)\right|^{2}+d, \quad \forall t \in[0, T),
$$

where $d$ is a positive constant. By Definition 1 we have $\left|\nabla V\left(\tilde{z}_{1 t}(t)\right)\right| \rightarrow \infty$ as $z_{1 t}(t)$ approaches the border of the constraints set, $\partial \mathcal{D}_{t}$. Therefore, if $\left|\tilde{z}_{1 t}(t)\right|$ grows, there exists a time $0<T^{*}<T$ such that $\dot{V}_{z}\left(\tilde{z}_{1 t}\left(T^{*}\right)\right) \leq 0$. The latter, in turn means that $V_{z}\left(\tilde{z}_{1 t}(t)\right)$ is bounded for all $t \in[0, T)$, which contradicts the initial assumption that the constraints are not respected. By resetting the initial conditions, the previous reasoning can be repeated for $t \geq T$. Therefore, the interval where $V_{z}\left(\tilde{z}_{1 t}(t)\right)$ is bounded can be extended to infinity. The boundedness of $V_{z}\left(\tilde{z}_{1 t}(t)\right)$ means, based on the definition of the BLF, that the constraints are always respected or, equivalently, that the set $\mathcal{D}_{t}$ is forward invariant.

\section{FORMATION CONTROL OF COOPERATIVE THRUST-PROPELLED UAVS WITH OUTPUT CONSTRAINTS}

In this section we use the previously-presented control method to solve a problem of robust consensus for multiagent constrained 3rd-order systems, which has relevance in certain robotics applications. The case-study consists in designing robust distributed controllers for position-consensusbased formation of multiple thrust-propelled UAVs under a set of realistic hypotheses. It is assumed that the drones are equipped with relative-measurement sensors, so the graph representing the interaction between the agents is directed.
Only directed-spanning-tree and directed-cycle topologies are considered but, alluding to Remark 5, we stress that the results in this section also apply to connected undirected graphs. Furthermore, the sensors are reliable only if "neighboring" agents remain within a certain range and, in order to guarantee the safety of the systems, these must also avoid collisions with one another. Finally, we assume that the agents are subject to bounded time-varying disturbances. Thus, this meaningful case-study shows both the applicability and the versatility of our framework. Its efficacy is illustrated via simulations.

\section{A. UAV's model and problem formulation}

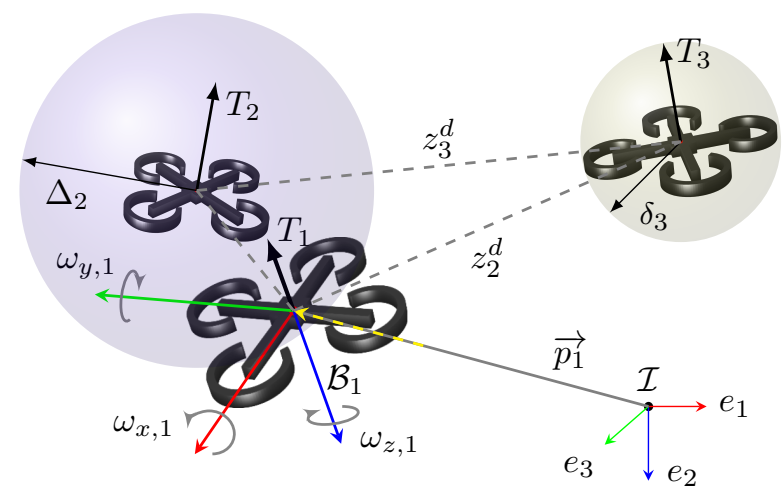

Fig. 2: Group of thrust propelled vehicles and Inertial frame

We consider a swarm of $N$ UAVs as illustrated in Fig. 2; each vehicle's motion being described by a so-called "mixed" model that consists in a second-order Cartesian dynamics equation on $\mathrm{E}(3)$ and a first-order attitude kinematics equation on $\mathrm{SO}(3)-$ see, e.g., [30], [31]. This underactuated model describes some commercial UAVs, which accept only thrust and angular rates as the control inputs.

The model for the $i$ th agent is given by the equations

$$
\begin{aligned}
\dot{p}_{i} & =v_{i} \\
\dot{v}_{i} & =-\frac{T_{i}}{m_{i}} \mathfrak{R}_{i} e_{3}+g e_{3}+\theta_{i, 2}(t) \\
\dot{\mathfrak{R}}_{i} & =\mathfrak{R}_{i} S\left(\omega_{i}\right),
\end{aligned}
$$

where $m_{i}$ is the mass of the quadrotor, $e_{3}=\left[\begin{array}{lll}0 & 0 & 1\end{array}\right]^{\top}$ is the unitary vector in the vertical direction of the inertial frame $\mathcal{I}$, $p_{i} \in \mathbb{R}^{3}$ and $v_{i} \in \mathbb{R}^{3}$ are respectively the inertial position and inertial velocity, $\mathfrak{R}_{i} \in \mathrm{SO}(3)$ is the rotation matrix of the body-fixed frame $\mathcal{B}_{i}$ with respect to $\mathcal{I}, g$ is gravity acceleration, and $\theta_{i, 2}: \mathbb{R}_{\geq 0} \rightarrow \mathbb{R}^{3}$ is an essentially bounded disturbance. The inputs are the thrust force produced by the propellers, $T_{i} \in \mathbb{R}$, and the angular rate of the vehicle $\omega_{i} \in \mathbb{R}^{3}$ in the body-fixed frame $\mathcal{B}_{i}$-see Figure 2 for an illustration.

The control goal is for the robots to achieve a predetermined formation in the three-dimensional space, centered at a point of non-predefined coordinates. More precisely, let the output of the multi-agent system be the relative position between pairs of connected agents. That is, in the way of (5)-(6), the edge states and error-edge states are defined as

$$
z_{1}:=\left[E^{\top} \otimes I_{3}\right] p
$$


and

$$
\tilde{z}_{1}=\left[E^{\top} \otimes I_{3}\right] p-z_{1}^{d},
$$

respectively, where $p^{\top}=\left[\begin{array}{lll}p_{1}^{\top} & \cdots & p_{N}^{\top}\end{array}\right] \in \mathbb{R}^{3 N}$ and $z_{1}^{d \top}=$ $\left[\begin{array}{lll}z_{1,1}{ }^{\top} & \cdots & z_{M, 1}^{d \top}\end{array}\right] \in \mathbb{R}^{3 M}$ are the relative displacements of the desired formation. Therefore, the control objective is that

$$
\begin{aligned}
\lim _{t \rightarrow \infty} \tilde{z}_{k, 1}(t)=0 & \forall k \leq M \\
\lim _{t \rightarrow \infty} v_{i}(t)=0 & \forall i \leq N .
\end{aligned}
$$

Next, let $\Delta_{k}$ denote the maximal distance between the nodes $i$ and $j$, such that the node $j$ has access to information from the node $i$ through the arc $e_{k}=(i, j)$. Similarly, let $\delta_{k}$ denote the minimal distance among neighbors such that collisions are avoided. Then, the connectivity and collision-avoidance constraints are encoded by the constraints set given in (3).

Robust formation problem with output constraints: Consider a multi-agent system composed of $N$ quadrotor UAVs with underactuated dynamics described by (58)-(59). Let the interactions of the vehicles be modeled by a connected undirected graph, a directed spanning tree or a directed cycle. Moreover, let the output inter-agent constraints be given by the set (3). Find distributed controllers $T_{i}$ and $\omega_{i}, i \leq N$, that, in the absence of disturbances, that is, with $\theta_{i, 2} \equiv 0$ for all $i \leq N$, achieve the objective (62) and render the constraints set (3) forward invariant, i.e., $z_{1}(0) \in \mathcal{D}$ implies that $z_{1}(t) \in \mathcal{D}$ for all $t \geq 0$. Furthermore, in the presence of disturbances, that is $\theta_{i, 2} \not \equiv 0$, the control law must render the formation practically input-to-state stable with respect to the disturbances and the set $\mathcal{D}$ in (3) forward invariant.

\section{B. Control approach}

The design builds on the method proposed in the previous sections, but we must start by applying an implementable feedback transformation to represent the system (58) in the form (1). Then, the control architecture follows a hierarchical approach that exploits the natural cascaded interconnection between the translational dynamics (58) and the rotational kinematics, (59) - see Fig. 3.

First, note that (58) may be assimilated to a second-order integrator

$$
\begin{aligned}
\dot{p}_{i} & =v_{i} \\
\dot{v}_{i} & =\zeta_{i}+\theta_{i, 2}(t),
\end{aligned}
$$

with

$$
\zeta_{i}:=-\frac{T_{i}}{m_{i}} \Re_{i} e_{3}+g e_{3} .
$$

Nonetheless, the implementation of a virtual controller for (63), through the input $\zeta_{i}$, is subject to the possibility of solving (64) for $T_{i}$, which is the actual control input. Because of the underactuation of (58), however, this is far from acquired. Indeed, note from (64) that the virtual input $\zeta_{i} \in \mathbb{R}^{3}$ cannot take an arbitrary value since $T_{i} \in \mathbb{R}$ and its direction are determined by the vehicle's orientation, $\mathfrak{R}_{i}$. In order to overcome the underactuation, we solve equation (64) dynamically, inspired by the distributed-backstepping approach in [31]. More precisely, we design the angular rates $\omega_{i}$ and an update law for the thrust $T_{i}$, so that $\zeta_{i}$ in (64) satisfies the dynamic equation

$$
\dot{\zeta}_{i}=u_{i}, \quad i \leq N,
$$

where $u_{i} \in \mathbb{R}^{3}$ is a new input. Note that now the system defined by (63) and (65) has the form (1) with $\rho=3$. Hence, in the sequel, in (65), $u_{i}$ is assumed to correspond to an output-constrained consensus control law designed as per the framework described in Section III.

Differentiating (64) with respect to time, and using (59), the left-hand side of (65) becomes

$$
-\frac{\dot{T}_{i}}{m_{i}} \mathfrak{R}_{i} e_{3}-\frac{T_{i}}{m_{i}} \Re_{i} S\left(\omega_{i}\right) e_{3}=u_{i} .
$$

Then, for a given $u_{i}$, we define $\nu_{i} \in \mathbb{R}^{3}$ as

$$
\nu_{i}:=u_{i}-\frac{c_{3}}{m_{i}} T_{i} \Re_{i} e_{3},
$$

where $c_{3}$ is a positive control gain. Next, replacing (67) into (66), we obtain

$$
\left[\left(\dot{T}_{i}+c_{3} T_{i}\right) \Re_{i}+T_{i} \Re_{i} S\left(\omega_{i}\right)\right] e_{3}=-m_{i} \nu_{i} .
$$

Left-multiplying by (the full-rank rotation matrix) $\mathfrak{R}_{i}^{\top}$, we see that the dynamic equation (68) is equivalent to

$$
\left[T_{i} \omega_{y i},-T_{i} \omega_{x i}, \dot{T}_{i}+c_{3} T_{i}\right]^{\top}=-m_{i} \Re_{i}^{\top} \nu_{i} .
$$

Now let $\tilde{\nu}_{i}:=\left[\begin{array}{lll}\tilde{\nu}_{i, x} & \tilde{\nu}_{i, y} & \tilde{\nu}_{i, z}\end{array}\right]^{\top}=\mathfrak{R}_{i}^{\top} \nu_{i}$. Then, (69) holds if the angular rates are set to

$$
\omega_{i}=\left[\begin{array}{lll}
\frac{m_{i} \tilde{\nu}_{i, y}}{T_{i}} & -\frac{m_{i} \tilde{\nu}_{i, x}}{T_{i}} & \omega_{z i}
\end{array}\right]^{\top},
$$

and the thrust is given by the update law

$$
\dot{T}_{i}=-c_{3} T_{i}-m_{i} \tilde{\nu}_{i, z} .
$$

Remark 10: Note that by transforming the UAV model (58) using (64)-(65), only the three translational dimensions are directly controlled. Therefore, only three of the four available inputs are needed to solve the formation problem. Indeed, note that from equation (69), the yaw component of the angular rate $\omega_{z i}$ is not needed for the control. Hence it may be considered as an additional degree of freedom and may be designed so that the vehicle follows a desired yaw trajectory.

Thus, after the previous transformation, the underactuated system (58) may be rewritten in the form (1), as desired, i.e.,

$$
\begin{aligned}
\dot{p}_{i} & =v_{i} \\
\dot{v}_{i} & =\zeta_{i}+\theta_{i, 2}(t) \\
\dot{\zeta}_{i} & =u_{i} .
\end{aligned}
$$

Furthermore, let $v^{\top}=\left[\begin{array}{lll}v_{1}^{\top} & \cdots & v_{N}^{\top}\end{array}\right] \in \mathbb{R}^{3 N}$ and $\zeta^{\top}=$ $\left[\zeta_{1}^{\top} \cdots \zeta_{N}^{\top}\right] \in \mathbb{R}^{3 N}$; using the transformation (61), the multiagent system in the reduced error-edge coordinates becomes

$$
\begin{aligned}
\dot{\tilde{z}}_{1 t} & =\left[E_{t}^{\top} \otimes I_{3}\right] v \\
\dot{v} & =\zeta+\theta_{2}(t) \\
\dot{\zeta} & =u .
\end{aligned}
$$




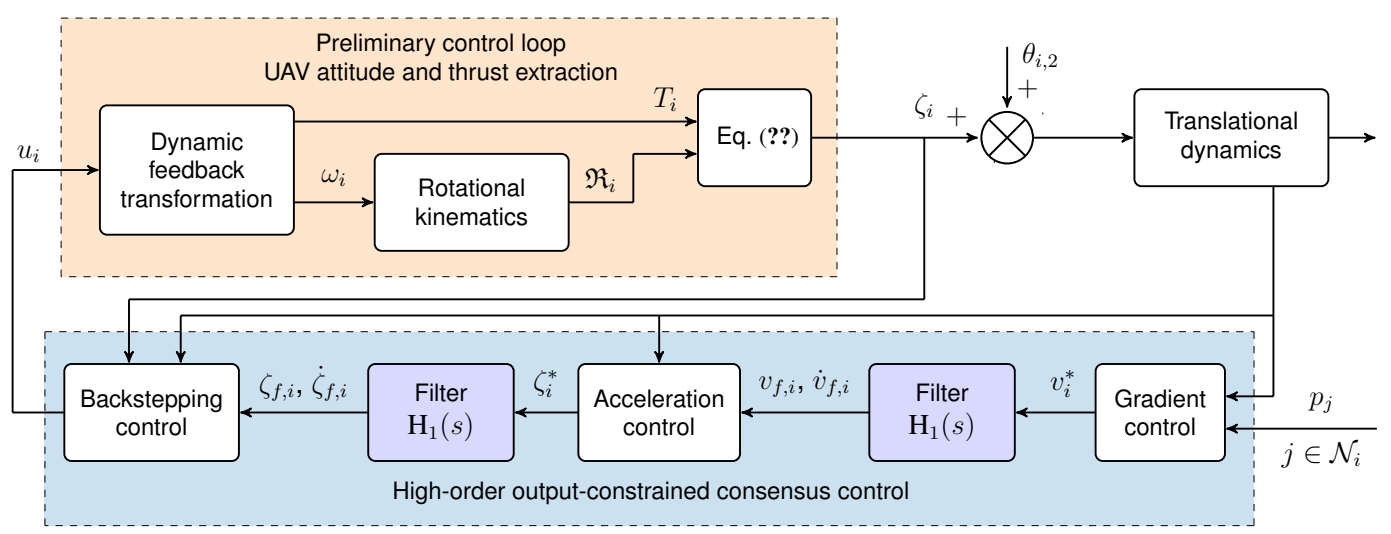

Fig. 3: Block diagram of the hierarchical control approach.

The transformed system (73) is in the form of (14) with $\rho=3$. To apply the robust control law (27), designed in the previous section for the constrained-consensus problem of high-order systems, we define the backstepping error variables

$$
\tilde{v}=v-v_{f} \quad \text { and } \quad \tilde{\zeta}=\zeta-\zeta_{f} .
$$

The filtered signals $v_{f}$ and $\zeta_{f}$ are the outputs of command filters given in (23), with inputs $v^{*}$ and $\zeta^{*}$, respectively, corresponding to the desired virtual controllers given by

$$
\begin{aligned}
& v^{*}:=-c_{1}\left[E_{\odot} \otimes I_{3}\right] \nabla W(\tilde{z}) \\
& \zeta^{*}:=-c_{2} \tilde{v}+\omega_{n} \alpha_{1,2},
\end{aligned}
$$

where $\nabla W(\tilde{z})$ is the gradient of the BLF defined in (18) and (17) with the weight recentered barrier function given by [41]

$$
\begin{aligned}
B_{k}\left(z_{k, 1}\right)= & \kappa_{1, k}\left[\ln \left(\frac{\Delta_{k}^{2}}{\Delta_{k}^{2}-\left|z_{k, 1}\right|^{2}}\right)-\ln \left(\frac{\Delta_{k}^{2}}{\Delta_{k}^{2}-\left|z_{k, 1}^{d}\right|^{2}}\right)\right] \\
& +\kappa_{2, k}\left[\ln \left(\frac{\left|z_{k, 1}\right|^{2}}{\left|z_{k, 1}\right|^{2}-\delta_{k}^{2}}\right)-\ln \left(\frac{\left|z_{k, 1}^{d}\right|^{2}}{\left|z_{k, 1}^{d}\right|^{2}-\delta_{k}^{2}}\right)\right], \\
\kappa_{1, k}:= & \frac{\delta_{k}^{2}}{\left|z_{k, 1}^{d}\right|^{2}\left(\left|z_{k, 1}^{d}\right|^{2}-\delta_{k}^{2}\right)}, \quad \kappa_{2, k}:=\frac{1}{\Delta_{k}^{2}-\left|z_{k, 1}^{d}\right|^{2}} .
\end{aligned}
$$

This function and its gradient are equal to zero at the desired formation configuration, i.e., $B_{k}\left(z_{k, 1}^{d}\right)=0, \nabla B_{k}\left(z_{k, 1}^{d}\right)=0$. Moreover it directly encodes the constraints in terms of the original edge-state $z_{k, 1}$, i.e., $B_{k}\left(z_{k, 1}\right) \rightarrow \infty$ as either $\left|z_{k, 1}\right| \rightarrow$ $\Delta_{k}$ or $\left|z_{k, 1}\right| \rightarrow \delta_{k}$-see Remark 3.

Then, akin to (27), the new input $u$ is given by

$$
u:=-c_{3} \tilde{\zeta}+\omega_{n} \alpha_{2,2}-\tilde{v} .
$$

After the developments in Section III and Theorem 1, the transformed controller (77), with (75)-(76) and (23), solves the robust formation problem with output constraints for system (72). However, a closer inspection shows that there is one more technical difficulty to circumvent. Note that, from (70), the dynamic solution to the equation (64) is valid if and only if $T_{i} \neq 0$. In order to address this additional constraint, we perform a control redesign which respects the control method and the stability analysis in Sections III-B and IV.
From (64), the condition $T_{i} \neq 0$ is satisfied if the filtered control $\zeta_{\text {if }}$ satisfies $\zeta_{\text {if }} \neq g e_{3}$, for all $i \leq N$. Let us modify the virtual control input $\zeta^{*}$ to

$$
\zeta^{*}:=\operatorname{sat}\left(-c_{2} \tilde{v}+\omega_{n} \alpha_{1,2}\right),
$$

where $\operatorname{sat}(\cdot)$ is a saturation function $\mathbb{R}^{N} \rightarrow \mathbb{R}^{N}$ defined element-wise, i.e., sat $(s)=\left[\sigma\left(s_{1}\right)^{\top} \cdots \sigma\left(s_{N}\right)^{\top}\right]^{\top}$, where, e.g., $\sigma\left(s_{i}\right)=\operatorname{sign}\left(s_{i}\right) \min \left\{\left|s_{i}\right|, \bar{\zeta}_{M}\right\}$, with $\bar{\zeta}_{M}<g$. Then, with the modified virtual control (78) as input, the over-damped command filter (23) with initial conditions $\alpha_{2,1}(0)=\zeta^{*}(0)$, $\alpha_{2,2}(0)=0$, guarantees that the output $\zeta_{i f}(t) \leq \bar{\zeta}_{M}<g$, and, consequently, that $T_{i}(t) \neq 0$ for all $t \geq 0$.

Remark 11: The virtual control $\zeta^{*}$ is saturated to ensure that the physical input, the thrust, $T_{i} \neq 0$. In addition, the control design also guarantees that $\zeta$ and the thrust $T_{i}$ satisfy pre-imposed bounds. This is significant because it implies that even though BLF-based controllers are not primarily designed to guarantee control-input constraints, the satisfaction of the latter may be accomplished using a dynamic controller, as done above.

Proposition 1: Under the Standing Assumption and for almost any initial conditions satisfying $z(0) \in \mathcal{D}$, except for a set of measure zero, there exists $\epsilon^{*}$, such that, for $\epsilon \in\left(0, \epsilon^{*}\right]$ where $\epsilon:=1 / \omega_{n}$, the control law (70)-(71) and (77), with (75), (78), and the command filters (23) with initial conditions set to $\alpha_{1,1}(0)=v^{*}(0), \alpha_{2,1}(0)=\zeta^{*}(0), \alpha_{1,2}(0)=\alpha_{2,2}(0)=0$, solves the robust formation problem with output constraints for system (58).

Proof: The proof follows the same arguments as the proof of Theorem 1. First, following the same arguments used in Section IV-A, the system (73) in closed-loop with (77) may be written in singular-perturbation form

$$
\begin{aligned}
\dot{\tilde{z}}_{1 t} & =-c_{1}\left[E_{t}^{\top} E_{\odot} \otimes I_{n}\right] \nabla W\left(\tilde{z}_{1}\right)+\left[E_{t}^{\top} \otimes I_{n}\right]\left[\tilde{v}+\tilde{\alpha}_{1,1}\right] \\
\dot{\tilde{v}} & =\operatorname{sat}\left(-c_{2} \tilde{v}+\omega_{n} \tilde{\alpha}_{1,2}\right)+\tilde{\zeta}+\tilde{\alpha}_{2,1}-\omega_{n} \tilde{\alpha}_{1,2}+\theta_{2}(t) \\
\dot{\tilde{\zeta}} & =-c_{3} \tilde{\zeta}-\tilde{v} \\
\epsilon \dot{\tilde{\alpha}} & =\tilde{A} \tilde{\alpha}-\epsilon \frac{\partial h(\xi)}{\partial \xi} \dot{\xi}, \quad \xi^{\top}=\left[\begin{array}{lll}
z_{1 t}^{\top} & \tilde{v}^{\top} & \tilde{\zeta}^{\top}
\end{array}\right] .
\end{aligned}
$$

Then, proceeding as in Section IV-B, to apply [28, Theorem 2], we need to show that the boundary layer system is asymptotically stable and that the reduced slow system is 
input-to-state stable with respect to the set $\mathcal{W} \times\{0\}^{2}$ and input $\theta_{i, 2}, i \leq N$.

Since $\tilde{A}$ is Hurwitz, the origin for the boundary-layer system (44) is exponentially stable. Now consider the reduced system

$$
\begin{aligned}
\dot{\tilde{z}}_{1 t} & =-c_{1}\left[E_{t}^{\top} E_{\odot} \otimes I_{n}\right] \nabla W\left(\tilde{z}_{1}\right)+\left[E_{t}^{\top} \otimes I_{n}\right] \tilde{v} \\
\dot{\tilde{v}} & =-\operatorname{sat}\left(c_{2} \tilde{v}\right)+\tilde{\zeta}+\theta_{2}(t) \\
\dot{\tilde{\zeta}} & =-c_{3} \tilde{\zeta}-\tilde{v} .
\end{aligned}
$$

From (55) the subsystem (79a) is input-to-state stable with respect to the set $\mathcal{W}$ and to the input $\tilde{v}$. Next, consider the subsystem (79b)-(79c). Let $\varepsilon_{u} \in(0,1)$ and define the Lyapunov function

$$
V_{2}(\tilde{v}, \tilde{\zeta})=\frac{\left(1+c_{3} \varepsilon_{u}\right)}{2}|\tilde{v}|^{2}+\frac{1}{2}|\tilde{\zeta}|^{2}+\varepsilon_{u} \tilde{\zeta}^{\top} \tilde{v}
$$

which is positive definite. Its derivative along (79b)-(79c) satisfies

$$
\begin{aligned}
& \dot{V}_{2}(\tilde{v}, \tilde{\zeta}) \leq-\left(1+\varepsilon_{u} c_{3}-\gamma_{v}\right)|\tilde{v}| \operatorname{sat}\left(c_{2}|\tilde{v}|\right) \\
& \quad-\left(c_{3}-\varepsilon_{u}-\frac{\varepsilon_{u}^{2}}{1+c_{3} \varepsilon_{u}}\right)|\tilde{\zeta}|^{2}+\frac{2 \varepsilon_{u}}{1+c_{3} \varepsilon_{u}}\left|\theta_{2}\right|^{2} .
\end{aligned}
$$

Hence, choosing $\gamma_{v}>0$ and $\varepsilon_{u}>0$ small enough so that

$$
\gamma_{2}:=c_{3}-\varepsilon_{u}-\frac{\varepsilon_{u}^{2}}{1+c_{3} \varepsilon_{u}}>0
$$

and $\gamma_{1}:=1+\varepsilon_{u} c_{3}-\gamma_{v}>0$, we have

$$
\dot{V}_{2}(\tilde{v}, \tilde{\zeta}) \leq-\gamma_{1}|\tilde{v}| \operatorname{sat}\left(c_{2}|\tilde{v}|\right)-\gamma_{2}|\tilde{\zeta}|^{2}+\gamma_{3}\left|\theta_{2}\right|^{2}
$$

where $\gamma_{3}:=2 \varepsilon_{u} /\left(1+c_{3} \varepsilon_{u}\right)$. The inequality (82) implies input-to-state stability of (79b)-(79c) with respect to the origin and to $\theta_{2}$. Using [29, Theorem 3.1] we conclude that, for all initial conditions $\xi(0)$ such that $z_{t}(0) \in \mathcal{D}_{t}$ and all essentially bounded inputs $\theta_{2}(t)$, the reduced system (79) is input-to-state stable with respect to $\mathcal{W}_{\Theta}:=\mathcal{W} \times\{0\}^{2}$ and to the input $\theta_{2}(t)$. Furthermore, $\mathcal{W}_{\Theta}$ qualifies as a $\mathcal{W}$-limit set for (79).

Now, since the boundary layer system is exponentially stable and the reduced system is input-to-state stable with respect to $\mathcal{W}_{\Theta}$ and $\theta_{2}(t)$, using Theorem 1 , we conclude that the controller (77), with (23), (75), and (78) solves the robust consensus problem with output constraints for system (73). That is, the controllers (70)-(71) solve the robust formation problem with output constraints for the system (58).

\section{A numerical example}

We illustrate the performance of the controller (70)-(71) via a numerical example consisting in the rendezvous of six UAVs, subject to inter-agent collision avoidance and connectivity restrictions. It is assumed that the measurement range of each agent is different and that they are equipped with proximity sensors. Hence, the network is modeled by a directed graph shown in Fig. 4. It is assumed, however, that only at the initial time the vehicles are interconnected, so the controller must preserve such connectivity.

The initial conditions and constraint parameters are presented in Table I. The desired formation corresponds to a hexagon and is determined by the desired relative position

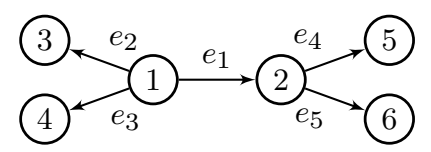

Fig. 4: Interaction topology: directed spanning tree

vector $z_{k, 1}^{d}=\left(z_{k, 1_{x}}^{d}, z_{k, 1_{y}}^{d}, z_{k, 1_{z}}^{d}\right)$, for each $k \leq 5$, set to $(1,0.5,0),(-1,1.5,0),(-1,0.5,0),(-2,1,0),(-1,0.5,0)$.

The saturation limit for the desired controller of the translational dynamics was set to $\bar{\zeta}_{M}=7 \mathrm{~m} / \mathrm{s}^{2}$, the controller gains to $c_{1}=1, c_{2}=0.8, c_{3}=3$, and the filter natural frequency to $\omega_{n}=350 \mathrm{rad} / \mathrm{s}$. The mass of each drone is $m_{i}=0.4 \mathrm{~kg}$.

TABLE I: Initial conditions and constraint parameters

\begin{tabular}{ccccccccc}
\hline $\begin{array}{c}\text { UAV } \\
\text { index }\end{array}$ & $\begin{array}{c}p_{x} \\
{[\mathbf{m}]}\end{array}$ & $\begin{array}{c}p_{y} \\
{[\mathbf{m}]}\end{array}$ & $\begin{array}{c}p_{z} \\
{[\mathbf{m}]}\end{array}$ & $\begin{array}{c}v_{x} \\
{[\mathbf{m} / \mathbf{s}]}\end{array}$ & $\begin{array}{c}v_{y} \\
{[\mathbf{m} / \mathbf{s}]}\end{array}$ & $\begin{array}{c}v_{z} \\
{[\mathbf{m} / \mathbf{s}]}\end{array}$ & $\begin{array}{c}\Delta_{k} \\
{[\mathbf{m}]}\end{array}$ & $\begin{array}{c}\delta_{k} \\
{[\mathbf{m}]}\end{array}$ \\
\hline 1 & 2.4 & 0 & -1 & 0.6 & -0.8 & 0 & 2.5 & 0.2 \\
\hline 2 & -0.58 & -0.9 & 0 & -0.3 & 0 & 0 & 3.4 & 0.2 \\
\hline 3 & 4 & 1.8 & 0 & 1.1 & 0.3 & 0 & 3.8 & 0.2 \\
\hline 4 & 5 & -2 & 0 & 0.1 & 0 & 0 & 3.5 & 0.2 \\
\hline 5 & -4.2 & -0.45 & 0 & 0 & 0 & 0 & 3.7 & 0.2 \\
\hline 6 & -2 & -4.2 & 2 & -0.8 & 0 & 0 & 4.2 & 0.2 \\
\hline
\end{tabular}

It is also assumed that the UAVs are subject to a disturbance modeled as a smoothed vanishing step, that is,

$$
\begin{aligned}
\theta_{i}(t) & =-\sigma_{i}(t)\left[\begin{array}{lll}
1 & 1 & 0
\end{array}\right]^{\top} \\
\sigma_{i}(t) & =\left\{\begin{array}{cll}
-0.6[\tanh (2(t-15))-1] & \text { if } \quad i \in\{3,5\} \\
0.6[\tanh (2(t-15))-1] & \text { if } \quad i=2 \\
0 & \text { if } i \in\{1,4,6\} .
\end{array}\right.
\end{aligned}
$$

The paths of each agent and the final desired formation are shown in Fig. 5. The trajectories of the inter-agent distances are presented in Fig. 6. It is also seen in the latter that the connectivity and collision avoidance constraints (dashed lines) are respected, even in the presence of the disturbance. Furthermore, as soon as the disturbance vanishes after 15 seconds, the agents converge to the desired static formation.

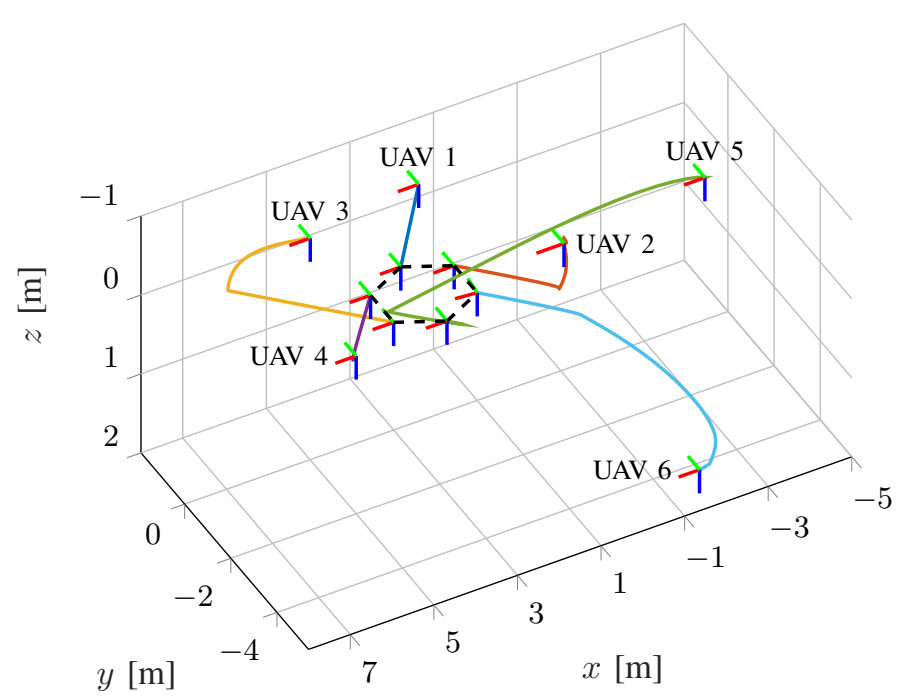

Fig. 5: Paths of the agents. The dashed black hexagon represents the final formation. 


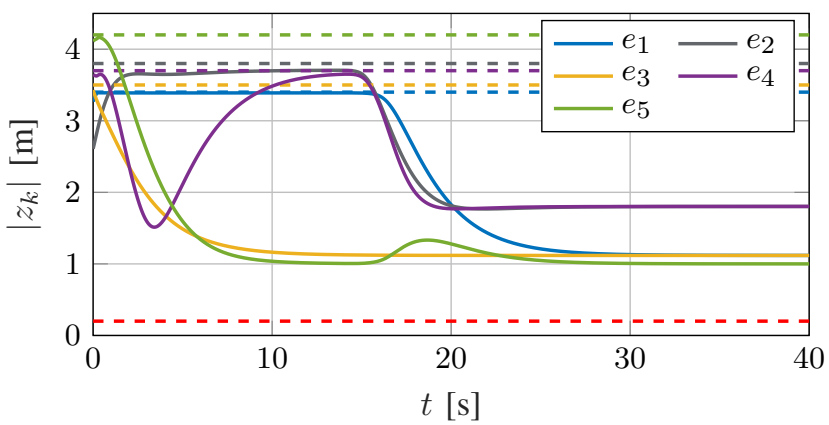

Fig. 6: Distances between neighbor UAVs. The dashed red line corresponds to the collision avoidance constraints and the dashed colored lines represent the connectivity constraints for the edge of the same color.

\section{CONCLUSIONS}

We presented a consensus control framework of broad applicability for systems of high relative degree in feedback form, subject to inter-agent constraints and interconnected over directed or undirected graphs. The design is based on the gradient of barrier Lyapunov functions and on the command filtered backstepping approach. It is important to emphasize that, beyond mere convergence to the consensus manifold, also robustness in the sense of practical input-to-state multistability with respect to external disturbances is established.

We also showed that our control framework is versatile in that it serves as basis for consensus control design of systems that are not, a priori, in the assumed strict-feedback form. In particular, we solved the rendezvous (open) problem for a group of UAVs subject to connectivity and inter-agent collision-avoidance constraints.

We believe that our theoretical results may be useful to approach other consensus-based problems in realistic settings for different types of dynamical systems. Current research focuses on consensus-tracking control with limited measurements and the generalization of our main theoretical statements to multiagent systems interconnected over arbitrary and time-varying directed topologies.

\section{APPENDIX I \\ STABILITY OF MULTIPLE INVARIANT SETS}

The following statements are adapted from the literature to the notation used in this paper. Theorem 3 is adapted from [28, Theorem 2], Definition 2 is adapted from from [29, Definition 2.7], and Theorem 4 from [29, Theorem 2.8].

Theorem 3: Consider a singularly perturbed system of the form (41). Assume that:

1) the reduced system (42) is input-to-state stable with respect to an acyclic $\mathcal{W}$-limit set $\mathcal{W}_{\Theta}$ and an input $\theta$;

2) the equilibrium $\tilde{\alpha}=0$ of the boundary layer system (44) is globally asymptotically stable.

Then, there exist a class $\mathcal{K} \mathcal{L}$ function $\beta_{\alpha}$ and a class $\mathcal{K}_{\infty}$ function $\eta_{\theta}$ and, for any pair $d_{1}, d_{2}>0$, there exists an $\epsilon^{*}>0$ such that, for any $\epsilon \in\left(0, \epsilon^{*}\right]$, any essentially bounded function $\theta(t)$, and any initial condition $\xi(0) \in \mathcal{D}_{t} \times \mathbb{R}^{n N(\rho-1)}$, and $\max \left\{|\xi(0)|_{\mathcal{W}_{\Theta}},|\tilde{\alpha}(0)|,\|\theta\|_{\infty},\|\dot{\theta}\|_{\infty}\right\} \leq d_{1}$, it holds that

$$
\begin{aligned}
|\tilde{\alpha}(t)| \leq \beta_{\alpha}\left(|\tilde{\alpha}(0)|, \frac{t}{\epsilon}\right)+d_{2} . \quad \forall t \geq 0 \\
\limsup _{t \rightarrow+\infty}|\xi(t)| \mathcal{W}_{\Theta} \leq \eta_{\theta}\left(\|\theta\|_{\infty}\right)+d_{2}
\end{aligned}
$$

Definition 2: A $\mathcal{C}^{1}$ function $V: \mathcal{M} \rightarrow \mathbb{R}_{\geq 0}$ is a practical ISS-Lyapunov function for a system $\dot{x}=f(x, \theta)$ if there exist $\mathcal{K}_{\infty}$ functions $\eta_{1}, \eta, \gamma$ and $q \geq 0$ such that, for all $x \in \mathcal{M}$ and all $\theta$, the following holds:

$$
\begin{aligned}
\eta_{1}\left(|x|_{\mathcal{W}}\right) & \leq V(x) \\
\nabla V(x)^{\top} f(x, \theta) & \leq-\eta\left(|x|_{\mathcal{W}}\right)+\gamma(|\theta|)+q .
\end{aligned}
$$

If (84) holds with $q=0$, then $V$ is said to be an ISS-Lyapunov function.

Theorem 4: Consider a system $\dot{x}=f(x, \theta)$ and an acyclic $\mathcal{W}$-limit set $\mathcal{W}$. Then, system $\dot{x}=f(x, \theta)$ is input-to-state stable with respect to input $\theta$ and to the set $\mathcal{W}$ if and only if it admits an ISS-Lyapunov function.

\section{REFERENCES}

[1] F. Chen, W. Ren et al., "On the control of multi-agent systems: A survey," Foundations and Trends ${ }^{\circledR}$ in Systems and Control, vol. 6, no. 4, pp. 339-499, 2019.

[2] R. Olfati-Saber, J. A. Fax, and R. M. Murray, "Consensus and cooperation in networked multi-agent systems," Proc. IEEE, vol. 95, no. 1, pp. 215-233, Jan. 2007.

[3] D. Mukherjee and D. Zelazo, "Robustness of consensus over weighted digraphs," IEEE Trans. Network Sc. Engg, vol. 6, no. 4, pp. 657-670, 2019.

[4] C. P. Bechlioulis and K. J. Kyriakopoulos, "Robust model-free formation control with prescribed performance and connectivity maintenance for nonlinear multi-agent systems," in 53rd IEEE Conf. Decis. Control, Los Angeles, CA, USA, 2014, pp. 4509-4514.

[5] A. Nikou, C. K. Verginis, and D. V. Dimarogonas, "Robust distancebased formation control of multiple rigid bodies with orientation alignment," IFAC-PapersOnLine, vol. 50, no. 1, pp. 15458-15463, 2017, 20th IFAC World Congress.

[6] W. Ren and R. Beard, Distributed Consensus in Multi-vehicle Cooperative Control: Theory and Applications, 1st ed. Springer Publishing Company, Incorporated, 2010.

[7] Y. Guo, Distributed cooperative control: emerging applications. John Wiley \& Sons, 2017.

[8] M. Mesbahi and M. Egerstedt, Graph theoretic methods in multiagent networks, ser. Princeton series in applied mathematics. Princeton: Princeton University Press, 2010, oCLC: ocn466341412.

[9] X. Wang, G. Wang, S. Li, and K. Lu, "Composite sliding-mode consensus algorithms for higher-order multi-agent systems subject to disturbances," IET Control Th. Appl., vol. 14, no. 2, pp. 291-303, 2019.

[10] C.-C. Hua, K. Li, and X.-P. Guan, "Leader-following output consensus for high-order nonlinear multiagent systems," IEEE Trans. Autom. Control, vol. 64, no. 3, pp. 1156-1161, 2018.

[11] H. Rezaee and F. Abdollahi, "Average consensus over high-order multiagent systems," IEEE Trans. Autom. Control, vol. 60, no. 11, pp. 30473052, 2015.

[12] Z. Zuo, B. Tian, M. Defoort, and Z. Ding, "Fixed-time consensus tracking for multiagent systems with high-order integrator dynamics," IEEE Trans. Autom. Control, vol. 63, no. 2, pp. 563-570, 2017.

[13] Z. Zhou and X. Wang, "Constrained consensus in continuous-time multiagent systems under weighted graph,” IEEE Trans. Autom. Control, vol. 63, no. 6, pp. 1776-1783, 2017.

[14] Y. Shang, "Resilient consensus in multi-agent systems with state constraints," Automatica, vol. 122, p. 109288, 2020.

[15] H. A. Poonawala and M. W. Spong, "Preserving strong connectivity in directed proximity graphs," IEEE Trans. Autom. Control, vol. 62, no. 9, pp. 4392-4404, Sep. 2017.

[16] E. Restrepo, A. Loría, I. Sarras, and J. Marzat, "Edge-based strict Lyapunov functions for consensus with connectivity preservation over directed graphs," Automatica, vol. 132, p. 109812, 2021. 
[17] J. Liu, C. Wang, and Y. Xu, "Distributed adaptive output consensus tracking for high-order nonlinear time-varying multi-agent systems with output constraints and actuator faults," J. of the Franklin Inst., vol. 357, no. 2, pp. 1090-1117, 2020.

[18] C. P. Bechlioulis and G. A. Rovithakis, "Decentralized robust synchronization of unknown high order nonlinear multi-agent systems with prescribed transient and steady state performance," IEEE Trans. Autom. Control, vol. 62, no. 1, pp. 123-134, 2016.

[19] J. Fu, G. Wen, Y. Lv, and T. Huang, "Barrier function based consensus of high-order nonlinear multi-agent systems with state constraints," in Int. Conf. Neural Information Processing, vol. 11954. Sydney, Australia: Springer, 2019, pp. 492-503.

[20] M. Hirche, P. N. Köhler, M. A. Müller, and F. Allgöwer, "Distributed model predictive control for consensus of constrained heterogeneous linear systems," in Proc. 59th IEEE Conf. Decis. Control (CDC), Jeju, Korea (South), 2020, pp. 1248-1253.

[21] S. Dubay and Y.-J. Pan, "Distributed mpc based collision avoidance approach for consensus of multiple quadcopters," in Proc. 2018 IEEE 14th Int. Conf. Control Autom. (ICCA), Anchorage, AK, USA, 2018, pp. $155-160$.

[22] D. A. Copp, K. G. Vamvoudakis, and J. P. Hespanha, "Distributed output-feedback model predictive control for multi-agent consensus," Syst. Control Lett., vol. 127, pp. 52-59, 2019.

[23] J. A. Marshall, M. E. Broucke, and B. A. Francis, "Formations of vehicles in cyclic pursuit," IEEE Trans. Autom. Control, vol. 49, no. 11, pp. 1963-1974, Nov 2004.

[24] K. P. Tee, S. S. Ge, and E. H. Tay, "Barrier Lyapunov functions for the control of output-constrained nonlinear systems," Automatica, vol. 45, no. 4, pp. 918-927, 2009.

[25] E. Rimon and D. E. Koditschek, "Exact robot navigation using artificial potential functions," IEEE Trans. Robot. Autom., vol. 8, no. 5, pp. 501518, Oct. 1992

[26] J. A. Farrell, M. Polycarpou, M. Sharma, and W. Dong, "Command filtered backstepping," IEEE Trans. Autom. Control, vol. 54, no. 6, pp. 1391-1395, 2009.

[27] D. Zelazo, A. Rahmani, and M. Mesbahi, "Agreement via the edge Laplacian," in Proc. 46th IEEE Conf. Decis. Control, New Orleans, LA, USA, 2007, pp. 2309-2314.

[28] P. Forni and D. Angeli, "Perturbation theory and singular perturbations for input-to-state multistable systems on manifolds," IEEE Trans. Autom. Control, vol. 64, no. 9, pp. 3555-3570, 2018.

[29] _ _ "Input-to-state stability for cascade systems with multiple invariant sets," Syst. Control Lett., vol. 98, pp. 97-110, 2016.

[30] M. Hua, T. Hamel, P. Morin, and C. Samson, "Introduction to feedback control of underactuated VTOL vehicles: A review of basic control design ideas and principles," IEEE Control Syst. Mag., vol. 33, no. 1, pp. 61-75, 2013.

[31] D. Lee, "Distributed backstepping control of multiple thrust-propelled vehicles on a balanced graph," Automatica, vol. 48, no. 11, pp. 29712977, 2012.

[32] A. Abdessameud, "Formation control of VTOL-UAVs under directed and dynamically-changing topologies," in Proc. 2019 American Control Conf. (ACC), Philadelphia, PA, USA, 2019, pp. 2042-2047.

[33] S. Bertrand, N. Guénard, T. Hamel, H. Piet-Lahanier, and L. Eck, "A hierarchical controller for miniature VTOL UAVs: Design and stability analysis using singular perturbation theory," Control Engg. Practice, vol. 19, no. 10, pp. 1099-1108, 2011.

[34] Y. Zou and Z. Meng, "Distributed hierarchical control for multiple vertical takeoff and landing UAVs with a distance-based network topology," Int. J. of Robust Nonlinear Control, vol. 29, no. 9, pp. 2573-2588, 2019.

[35] J. Ghommam, L. F. Luque-Vega, and M. Saad, "Distance-based formation control for quadrotors with collision avoidance via Lyapunov barrier functions," Int. J. Aerospace Engg., 2020.

[36] M. Ji and M. Egerstedt, "Distributed coordination control of multiagent systems while preserving connectedness," IEEE Trans. Robot., vol. 23 , no. 4, pp. 693-703, Aug. 2007.

[37] D. Boskos and D. V. Dimarogonas, "Robustness and invariance of connectivity maintenance control for multiagent systems," SIAM $J$. Control and Optimization, vol. 55, no. 3, pp. 1887-1914, 2017.

[38] E. Restrepo, A. Loría, I. Sarras, and J. Marzat, "Stability and robustness of edge-agreement-based consensus protocols for undirected proximity graphs," Int. J. Control, pp. 1-9, 2020.

[39] K. P. Tee and S. S. Ge, "Control of state-constrained nonlinear systems using integral barrier Lyapunov functionals," in Proc. 51st IEEE Conf. Decis. Control (CDC). Maui, HI, USA: IEEE, 2012, pp. 3239-3244.
[40] C. Feller and C. Ebenbauer, "Weight recentered barrier functions and smooth polytopic terminal set formulations for linear model predictive control," in Proc. 2015 American Control Conf. (ACC). Chicago, IL, USA: IEEE, 2015, pp. 1647-1652.

[41] E. Restrepo, "Coordination control of autonomous robotic multi-agent systems under constraints," Ph.D. dissertation, Univ Paris Saclay, Gif sur Yvette, France, 2021, https://tel.archives-ouvertes.fr/.

[42] Z. Zeng, X. Wang, and Z. Zheng, "Nonlinear consensus under directed graph via the edge Laplacian," in Proc. 26th Chinese Control Decis. Conf. (2014 CCDC). Changsha, China: IEEE, May 2014, pp. 881886.

[43] W. Dong, J. A. Farrell, M. M. Polycarpou, V. Djapic, and M. Sharma, "Command filtered adaptive backstepping," IEEE Trans. Control Syst. Technol., vol. 20, no. 3, pp. 566-580, 2011.

[44] P. V. Kokotović and H. J. Sussmann, "A positive real condition for global stabilization of nonlinear systems," Syst. Control Lett., vol. 13, no. 4, pp. 125-133, 1989.

[45] R. Sepulchre, M. Janković, and P. V. Kokotović, "Recursive designs and feedback passivation," in Systems and control in the twenty-first century. Springer, 1997, pp. 313-326.

[46] H. K. Khalil, Nonlinear systems; 2nd ed. Upper Saddle River, NJ: Prentice-Hall, 1996

[47] P. Monzón and R. Potrie, "Local and global aspects of almost global stability," in Proc. 45th IEEE Conf. Decis. Control. San Diego, CA USA: IEEE, 2006, pp. 5120-5125.

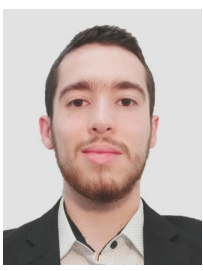

Esteban Restrepo obtained his BSc degree on Mechatronics engineering from Universidad EIA, Envigado, Colombia and from ENSAM, Paris, France in 2017. He obtained his MSc in Robotc Systems Engineering from ENSAM, Paris, France in 2018, receiving the Silver Medal honorary distinction. Shortly before the acceptance of this paper, he obtain the $\mathrm{PhD}$ degree in Automatic Control at the University Paris-Saclay, France and accepted a post-doctoral position at KTH, Stockholm, Sweden. His research interests include multi-agent systems and nonlinear control with application to autonomous robots and aerospace systems.

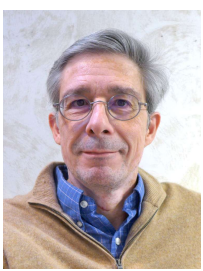

Antonio Loría obtained his BSc degree on Electronics Engineering from ITESM, Monterrey, Mexico in 1991 and his MSc and $\mathrm{PhD}$ degrees in Control Engg. from UTC, France in 1993 and 1996, respectively. He has the honour of holding a researcher position at the French National Centre of Scientific Research (CNRS) since January 1999 (Senior Researcher since 2006). He has co-authored over 250 publications on control systems and stability theory and he served as AE for IEEE Trans. Automat. Control, IEEE Trans. Control Syst. Techn., IEEE Control Syst. Lett., and the IEEE Conf. Editorial Board, as well as other jounrals on Automatic Control.

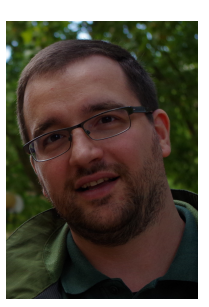

Dr. Ioannis Sarras (M '09) graduated from the Automation Engineering Department of the Technological Education Institute (T.E.I.) of Piraeus, Greece, in 2004. He obtained his MSc in Control Theory from the University of Paul Sabatier in 2006 and the PhD degree on Automatic Control from the University of Paris-Sud in 2010. Between 2010 and 2017 he was a research associate with IFPEn, ULB, MINES ParisTech and ONERA. Since 2017 he has been a Research Engineer at ONERA-The French Aerospace Lab. He has co-authored over 60 papers in peer-reviewed international journals and conferences. His research activities are in the fields of nonlinear control and observer designs for single- or multi-agent systems with emphasis on aerospace applications.

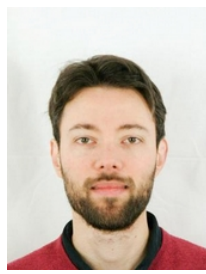

Julien Marzat graduated as an engineer from ENSEM (INPL Nancy) in 2008 and completed his PhD Thesis in 2011 and Habilitation in 2019, both from University ParisSaclay. He is currently a Research Scientist at ONERA, where his research interests include guidance, control and fault diagnosis for autonomous robots and aerospace systems. 